Discrete Comput Geom 38:479-512 (2007)

DOI: $10.1007 / \mathrm{s} 00454-007-1356-1$

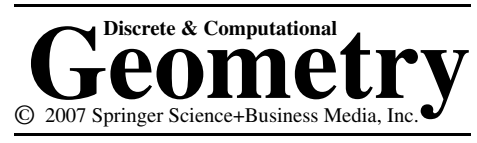

\title{
Stability of Critical Points with Interval Persistence*
}

\author{
Tamal K. Dey and Rephael Wenger \\ Department of Computer Science and Engineering, The Ohio State University, \\ Columbus, OH 43210, USA \\ \{tamaldey,wenger\}@cse.ohio-state.edu
}

\begin{abstract}
Scalar functions defined on a topological space $\Omega$ are at the core of many applications such as shape matching, visualization and physical simulations. Topological persistence is an approach to characterizing these functions. It measures how long topological structures in the sub-level sets $\{x \in \Omega: f(x) \leq c\}$ persist as $c$ changes. Recently it was shown that the critical values defining a topological structure with relatively large persistence remain almost unaffected by small perturbations. This result suggests that topological persistence is a good measure for matching and comparing scalar functions. We extend these results to critical points in the domain by redefining persistence and critical points and replacing sub-level sets $\{x \in \Omega: f(x) \leq c\}$ with interval sets $\{x \in \Omega: a \leq f(x)<b\}$. With these modifications we establish a stability result for critical points. This result is strengthened for maxima that can be used for matching two scalar functions.
\end{abstract}

\section{Introduction}

A scalar field is a scalar function $f: \Omega \rightarrow \mathbb{R}$ defined on some topological space $\Omega$. Examples of scalar fields are fluid pressure in computational fluid dynamics simulations, temperature in oceanographic or atmospheric studies, and density in medical CT or MRI scans. A level set of a scalar field is a set of points with the same scalar value, i.e., $\{x \in \Omega: f(x)=c\}$. One way of deriving quantitative information about scalar fields is by studying the topological structures of its level sets or the sub-level sets, such as $\{x \in \Omega: f(x) \leq c\}$. The mathematical field of Morse Theory is the study of these topological structures.

Among the most basic problems on scalar fields is simplifying a scalar field for compact representation, identifying important features in a scalar field, and characterizing the essential structure of a scalar field. Extracting and representing the topological

* Research partly supported by NSF Grants DMS-0310642 and CCF-0635008. 
structure of the level sets is one way of approaching all these problems. However, this topological structure may contain "small" topological features which are insignificant or caused by noise. Small topological features should be removed in simplification and ignored in characterizing essential structure or identifying important features. How does one determine which topological features are small?

Edelsbrunner et al. [8] introduced the notion of topological persistence. As $c \in \mathbb{R}$ increases, topological features appear and disappear in the sub-level set $\{x \in \Omega: f(x) \leq c\}$. If a topological feature appears at "time" $a$ and disappears at "time" $b$, then its persistence is the difference, $b-a$, between these two times. Edelsbrunner et al. [8] use homology groups over $\mathbb{Z} / 2 \mathbb{Z}$ to define topological features. Zomorodian and Carlsson [13] generalized the theory of topological persistence to homology groups over any fields.

Topological persistence gives an approach to comparing scalar fields. Two fields are similar if they have matching topological features with approximately the same persistence. This approach to comparing fields makes sense only if persistence remains stable under relatively small perturbations of the scalar fields. Cohen-Steiner et al. [4] proved that "large" persistence values remain almost unaffected. More precisely, let scalar field $\hat{f}: \Omega \rightarrow \mathbb{R}$ be a small perturbation of field $f: \Omega \rightarrow \mathbb{R}$ (i.e., $|\hat{f}(x)-f(x)| \leq \delta$ for all $x \in \Omega$ ). If $f$ has a topological structure with relatively large persistence which appears at $a$ and disappears at $b$, then $\hat{f}$ has a corresponding topological structure which appears around $a$ and disappears around $b$.

Critical Point Stability. The result of Cohen-Steiner et al. [4] showed that the critical values for structures with large persistence remain stable under small perturbations of the scalar field. Scalar fields also have critical points, points in the domain which change the topological structure of the level sets. It is natural to ask if critical points for structures with large persistence remain stable under perturbations of the field. If two scalar fields are close, then are their significant critical points "close"?

At the very onset, the problem of stability for critical points appears almost hopeless. Consider functions $f$ and $\hat{f}$ in Fig. 1 where $|f(x)-\hat{f}(x)|<\delta$ for all $x \in \Omega$. The maxima, $p$ and $p^{\prime}$, of $f$ and $\hat{f}$, respectively, can be made arbitrarily far apart even as $\delta$ is made arbitrarily small. So, there is no result for stability of critical points in terms of Hausdorff distances as considered by Cohen-Steiner et al. [4] for critical values.

One main contribution of this paper is to overcome this difficulty by defining a new notion of stability for the critical points. Instead of using a metric in the domain, we use the range to determine neighborhoods of points. These neighborhoods help define the

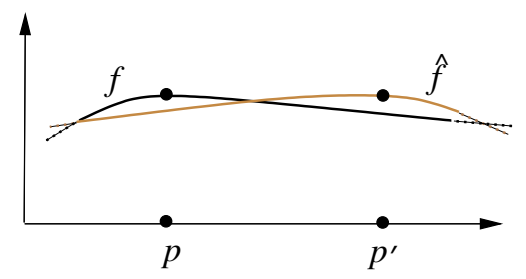

Fig. 1. The maximum $p$ for a real-valued function $f$ has moved by a large distance even for an arbitrarily close approximant $\hat{f}$. 
stability. An $\left(\eta_{1}, \eta_{2}\right)$-neighborhood of a point $p$ is the pathwise connected component of $\left\{x \in \Omega: f(p)-\eta_{1} \leq f(x) \leq f(p)+\eta_{2}\right\}$ containing $p$. A point which is in this neighborhood for small values of $\eta_{1}$ and $\eta_{2}$ is "close" to $p$. Note that points $p$ and $p^{\prime}$ in Fig. 1 are close in this sense. We show that if $p$ destroys a "persistent homology element" in $f$, then an $\left(\eta_{1}, \eta_{2}\right)$-neighborhood of $p$ contains a point $p^{\prime}$ which destroys a persistent homology element in $\hat{f}$. The values of $\eta_{1}$ and $\eta_{2}$ depend upon the persistence of the homology element and the difference $\delta$ between $f$ and $\hat{f}$. Theorem 3 states this result formally. Although this result relates critical points for functions under perturbations, it does not match them. To construct a matching of critical points, we need each destroying critical point of $\hat{f}$ to be in the neighborhood of only one critical point of $f$. We establish this stronger result for local maxima of functions on manifolds.

Interval Persistence. As with the critical values, not all critical points can be stable under perturbations. One may expect that only those critical points responsible for persistent homology structures remain stable. Therefore, we need a notion of persistence for critical points. Instead of using topological persistence as in [4], we use interval persistence for this purpose. There are two reasons to introduce this new notion of persistence. First, it can certify more critical points as persistent than the original topological persistence does. Second, interval persistence gives a natural setting for our critical point stability result in Theorem 3 . We illustrate the first point with an example below. The second point is discussed in Section 4 after Theorem 3.

Let $f(x)$ be the $z$-coordinate of any point $x$ on the surface in $\mathbb{R}^{3}$ shown in Fig. 2. There are eight critical points $\left\{p_{i}, i=0, \ldots, 7\right\}$. For sub-level sets, point $p_{1}$ creates the homology element $\left[c_{2}\right]$ generated by the cycle $c_{2}$. Homology element $\left[c_{2}\right]$ is destroyed by $p_{2}$. Thus, $p_{1}$ is paired with $p_{2}$. Similarly, point $p_{3}$ creates the homology element $\left[c_{1}\right]$ which is destroyed by $p_{4}$, so $p_{3}$ is paired with $p_{4}$. The critical points $p_{0}, p_{5}, p_{6}$, and $p_{7}$ create homology elements that are never destroyed and so these critical points remain unpaired by topological persistence.

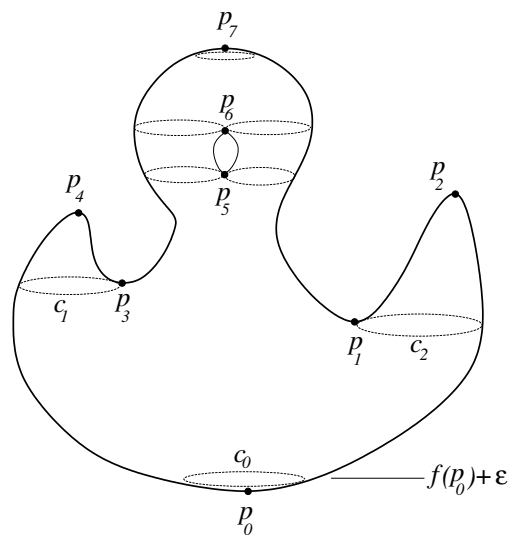

Fig. 2. Critical points $p_{0}, p_{1}, \ldots, p_{7}$. Pairs under topological persistence are $\left(p_{1}, p_{2}\right)$ and $\left(p_{3}, p_{4}\right)$. Pairs under interval persistence are $\left(p_{1}, p_{2}\right),\left(p_{3}, p_{4}\right),\left(p_{0}, p_{7}\right)$, and $\left(p_{5}, p_{6}\right)$. 
On the other hand, if we consider the interval set $\left\{x \in \Omega: f\left(p_{0}\right)<f(x)<f\left(p_{7}\right)\right\}$, the homology element $\left[c_{0}\right]$ starts just after $p_{0}$ and ends at $p_{7}$ (note that the interval set does not include $p_{0}$ or $p_{7}$ ). Interval persistence pairs $p_{0}$ with $p_{7}$. The interval set $\left\{x \in \Omega: f\left(p_{5}\right)<f(x)<f\left(p_{6}\right)\right\}$ has two connected components which get joined by $p_{5}$ and $p_{6}$. Again, interval persistence pairs $p_{5}$ with $p_{6}$. We give formal definitions and explanations of interval persistence and this pairing in Section 2.6.

Agarwal et al. in [1] proposed a different extension of the topological persistence pairings using Reeb graphs. This pairing is deduced only for 2-manifolds as opposed to the broader class of topological spaces covered by interval persistence.

\section{Definitions and Assumptions}

\subsection{Homology Groups}

For a topological space $X$, the $k$ th homology group $H_{k}(X)$ is an algebraic encoding of the connectivity of $X$ in the $k$ th dimension. For a good exposition on homology groups we refer to Hatcher [9]. We use singular homology which is more general than simplicial or cellular homology. We also use reduced homology groups, usually represented as $\tilde{H}_{k}(X)$. Groups $H_{k}(X)$ and $\tilde{H}_{k}(X)$ are exactly the same for all $k>0$, and their rank differs by one when $k$ equals zero. Because we use reduced homology almost everywhere in this paper, we drop the " " mark and represent $\tilde{H}_{k}(X)$ as $H_{k}(X)$, except where otherwise noted.

Although homology groups are defined for coefficients drawn from any ring, we consider only fields such as $\mathbb{R}, \mathbb{Q}, \mathbb{Z}_{p}=\mathbb{Z} / p \mathbb{Z}$ for a prime $p$ as in the previous works [4], [13]. Over fields the homology groups are vector spaces and the rank of $H_{k}(X)$, denoted $\beta_{k}(X)$, is called the $k$ th Betti number of $X$.

A continuous map $f: X \rightarrow Y$ between two topological spaces $X$ and $Y$ induces

a homomorphism, say $f_{*}$, between their homology groups, $H_{k}(X) \stackrel{f_{*}}{\rightarrow} H_{k}(Y)$. This property is carried over the composition of maps, that is, $(f \circ g)_{*}=f_{*} \circ g_{*}$. In our case, the maps between spaces will be inclusion maps. This means, if $X \subseteq Y$, we will consider the map $H_{k}(X) \stackrel{i_{*}}{\rightarrow} H_{k}(Y)$ where $i_{*}$ is induced by the inclusion map $i: X \rightarrow Y$.

\subsection{Interval Sets}

We use the following notation to define the interval sets bounded by the level sets of a function. For $a, b \in \mathbb{R}$ and functions $f$ and $g$, let

$$
\begin{aligned}
& F_{a}^{b}=\{x \in \Omega: a<f(x)<b\} \quad \text { and } \\
& G_{a}^{b}=\{x \in \Omega: a<g(x)<b\} .
\end{aligned}
$$

In our results and proofs we need the space $F_{a}^{b}$ and $G_{a}^{b}$ closed at the bottom. So, we define

$$
\underline{F}_{a}^{b}=\{x \in \Omega: a \leq f(x)<b\} \quad \text { and }
$$




$$
\underline{G}_{a}^{b}=\{x \in \Omega: a \leq g(x)<b\} .
$$

Notice that $a$ could be $-\infty$ and $b$ could be $\infty$. With these notations, the $\left(\eta_{1}, \eta_{2}\right)$ neighborhood of a point $p$ is the connected component of $\operatorname{cl}\left(F_{f(p)-\eta_{1}}^{f(p)+\eta_{2}}\right)$ containing $p$. Here cl denotes the closure.

\subsection{Critical Values and Points}

Intuitively, a critical value is a value at which the homology of an interval set changes. A critical point is a point on the boundary of an interval set whose addition to that interval set changes its homology. We give the following formal definitions:

Definition 1. Value $b \in \mathbb{R}$ is $H_{k}$-critical for the interval sets of $f: \Omega \rightarrow \mathbb{R}$ if $H_{k}\left(F_{a}^{b}\right) \rightarrow$ $H_{k}\left(F_{a}^{b} \cup f^{-1}(b)\right)$ is not an isomorphism for some $a<b$ or if $H_{k}\left(F_{b}^{c}\right) \rightarrow H_{k}\left(F_{b}^{c} \cup f^{-1}(b)\right)$ is not an isomorphism for some $c>b$.

Definition 2. Point $p \in \Omega$ is $H_{k}$-critical for the interval sets of $f: \Omega \rightarrow \mathbb{R}$ if $H_{k}\left(F_{a}^{f(p)}\right) \rightarrow$ $H_{k}\left(F_{a}^{f(p)} \cup\{p\}\right)$ is not an isomorphism for some $a<f(p)$ or if $H_{k}\left(F_{f(p)}^{c}\right) \rightarrow$ $H_{k}\left(F_{f(p)}^{c} \cup\{p\}\right)$ is not an isomorphism for some $c>f(p)$.

Note that in both definitions, $a$ could be $-\infty$ or $c$ could be $\infty$. A value or point can be $H_{k}$-critical for different values of $k$.

Cohen-Steiner et al. [4] give a similar definition for critical values. However, the definition of Cohen-Steiner et al. uses only sub-level sets instead of interval sets. Point $p \in \Omega$ is an $H_{k}$-critical point for the sub-level sets of $f$ if $H_{k}\left(F_{-\infty}^{f(p)}\right) \rightarrow H_{k}\left(F_{-\infty}^{f(p)} \cup\{p\}\right)$ is not an isomorphism for some integer $k \geq 0$.

As shown in Fig. 3, $H_{k}$-critical points for interval sets are not quite equivalent to $H_{k}$ critical points for sub-level sets. In Fig. 3 the mapping $H_{k}\left(F_{-\infty}^{f(p)}\right) \rightarrow H_{k}\left(F_{-\infty}^{f(p)} \cup\{p\}\right)$ is an isomorphism for any integer $k$. On the other hand, $H_{0}\left(F_{f(p)}^{b}\right) \rightarrow H_{0}\left(F_{f(p)}^{b} \cup\{p\}\right)$ is not an isomorphism and so $p$ is a critical point for interval sets.

Of course, if we replace function $f$ in Fig. 3 by function $-f$ then the mapping $H_{0}\left(F_{-\infty}^{-f(p)}\right) \rightarrow H_{0}\left(F_{-\infty}^{-f(p)} \cup\{p\}\right)$ is no longer an isomorphism. Thus, $p$ is an $H_{0}$-critical point for the sub-level sets of the function $-f$.

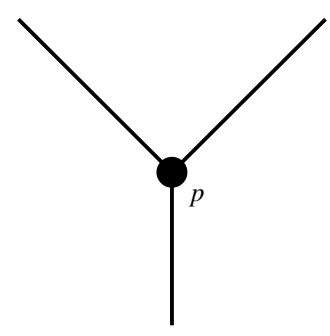

Fig. 3. Function $f$ is the height function in the $y$-direction. The mapping $H_{k}\left(F_{-\infty}^{f(p)}\right) \rightarrow H_{k}\left(F_{-\infty}^{f(p)} \cup\{p\}\right)$ is an isomorphism for any $k \geq 0$ but $H_{0}\left(F_{f(p)}^{b}\right) \rightarrow H_{0}\left(F_{f(p)}^{b} \cup\{p\}\right)$ is not for any $b>f(p)$. 
Figure 3 illustrates the following relationship between critical points for interval sets and critical points for sub-level sets. A point which is critical for the interval sets of $f$ is either critical for the sub-level sets of $f$ or critical for the sub-level sets of $-f$ or both (Theorem 1.)

To prove Theorem 1, we first give the following relationship between $H_{k}\left(F_{a}^{f(p)}\right) \rightarrow$ $H_{k}\left(F_{a}^{f(p)} \cup\{p\}\right)$ and $H_{-\infty}\left(F_{a}^{f(p)}\right) \rightarrow H_{k}\left(F_{-\infty}^{f(p)} \cup\{p\}\right)$. The proof is based on homological algebra and is left to Appendix B.

Lemma 1. Let a be some value less than $f(p)$. Mapping $H_{k}\left(F_{a}^{f(p)}\right) \rightarrow H_{k}\left(F_{a}^{f(p)} \cup\right.$ $\{p\})$ is an isomorphism for all integers $k \geq 0$ if and only if $H_{k}\left(F_{-\infty}^{f(p)}\right) \rightarrow H_{k}\left(F_{-\infty}^{f(p)} \cup\{p\}\right)$ is an isomorphism for all integers $k \geq 0$.

Note that Lemma 1 does not hold if $k$ is fixed. For instance, in Figure 2 the mapping $H_{1}\left(F_{-\infty}^{f\left(p_{7}\right)}\right) \rightarrow H_{1}\left(F_{-\infty}^{f\left(p_{7}\right)} \cup\left\{p_{7}\right\}\right)$ is an isomorphism while the mapping $H_{1}\left(F_{f\left(p_{6}\right)}^{f\left(p_{7}\right)}\right) \rightarrow$ $H_{1}\left(F_{f\left(p_{6}\right)}^{f\left(p_{7}\right)} \cup\left\{p_{7}\right\}\right)$ is not. There is no contradiction to Lemma 1 , since $H_{2}\left(F_{-\infty}^{f\left(p_{7}\right)}\right) \rightarrow$ $H_{2}\left(F_{-\infty}^{f\left(p_{7}\right)} \cup\left\{p_{7}\right\}\right)$ is not an isomorphism.

Theorem 1 follows directly from Lemma 1.

Theorem 1. For some $k \geq 0$, a point $p$ is $H_{k}$-critical for the interval sets of $f: \Omega \rightarrow \mathbb{R}$ if and only if, for some $j \geq 0, p$ is $H_{j}$-critical for the sub-level sets of $f$ or $-f$ (or both).

Proof. A point $p$ is an $H_{k}$-critical point for the interval sets of $f$ if $H_{k}\left(F_{a}^{f(p)}\right) \rightarrow$ $H_{k}\left(F_{a}^{f(p)} \cup\{p\}\right)$ is not an isomorphism for some $a<f(p)$ or if $H_{k}\left(F_{f(p)}^{c}\right) \rightarrow H_{k}\left(F_{f(p)}^{c} \cup\right.$ $\{p\})$ is not an isomorphism for some $c>f(p)$. Since $a$ can be $-\infty$, a point which is $H_{k}$-critical for the sub-level sets of $f$ is $H_{k}$-critical for the interval sets of $f$. Since $c$ can be $\infty$, a point which is $H_{k}$-critical for the sub-level sets of $-f$ is also $H_{k}$-critical for the interval sets of $f$. Thus, if $p$ is $H_{k}$-critical for the sub-level sets of $f$ or of $-f$, then $p$ is $H_{k}$-critical for the interval sets of $f$.

Assume $p$ is $H_{k}$-critical for the interval sets of $f$. By definition, either mapping $H_{k}\left(F_{a}^{f(p)}\right) \rightarrow H_{k}\left(F_{a}^{f(p)} \cup\{p\}\right)$ is not an isomorphism, $a<f(p)$, or $H_{k}\left(F_{f(p)}^{c}\right) \rightarrow$ $\left.H_{k}\left(F_{f(p)}^{c}\right) \cup\{p\}\right)$ is not an isomorphism, $c>f(p)$ (or both). By Lemma 1, if $H_{k}\left(F_{a}^{f(p)}\right) \rightarrow$ $H_{k}\left(F_{a}^{f(p)} \cup\{p\}\right)$ is not an isomorphism, then $H_{j}\left(F_{-\infty}^{f(p)}\right) \rightarrow H_{j}\left(F_{-\infty}^{f(p)} \cup\{p\}\right)$ is not an isomorphism for some integer $j$. Similarly, if $\left.H_{k}\left(F_{f(p)}^{c}\right) \rightarrow H_{k}\left(F_{f(p)}^{c}\right) \cup\{p\}\right)$ is not an isomorphism, then $H_{j}\left(F_{f(p)}^{\infty}\right) \rightarrow H_{j}\left(F_{f(p)}^{\infty} \cup\{p\}\right)$ is not an isomorphism for some integer $j$. If $H_{j}\left(F_{-\infty}^{f(p)}\right) \rightarrow H_{j}\left(F_{-\infty}^{f(p)} \cup\{p\}\right)$ is not an isomorphism, then $p$ is $H_{j}$-critical for the sub-level sets of $f$. If $H_{j}\left(F_{f(p)}^{\infty}\right) \rightarrow H_{k}\left(F_{f(p)}^{\infty} \cup\{p\}\right)$ is not an isomorphism, then $p$ is $H_{j}$-critical for the sub-level sets of $-f$.

For the rest of this paper, all critical points are $H_{k}$-critical for interval sets, unless otherwise noted. 


\subsection{Destruction}

Let $\Omega$ be a topological space. We define the destruction of homology elements in sets by critical points and other sets in general.

Definition 3. For $X \subseteq \Omega$ and $Y \subseteq \Omega$, set $Y$ destroys non-zero $h \in H_{k}(X)$ if the image of $h$ under the mapping $H_{k}(X) \rightarrow H_{k}(X \cup Y)$ is zero. In particular, if $q$ is a point in $\Omega$, point $q$ destroys non-zero $h \in H_{k}(X)$ if the image of $h$ under the mapping $H_{k}(X) \rightarrow H_{k}(X \cup\{q\})$ is zero.

The above definition does not apply to points that are not in the closure of $X$ though we encounter this situation repeatedly. We would like to say that a cycle generated in a level set is destroyed by a point disjoint from the level set. So, we extend the definition of destruction slightly.

Definition 4. If $X \subseteq Z \subseteq \Omega$ and $Y \subseteq \Omega$, then we say that $Y$ destroys the image of $h_{x} \in H_{k}(X)$ in $H_{k}(Z)$, if $h_{z} \in H_{k}(Z)$ is the image of $h_{x}$ under the mapping $H_{k}(X) \rightarrow$ $H_{k}(Z)$ and $h_{z}$ is non-zero and $Y$ destroys $h_{z}$.

We apply this definition repeatedly where $X$ is some level set $f^{-1}(a)$ and $Y$ is a point. For brevity, we say that point $q$ destroys $h \in H_{k}\left(f^{-1}(a)\right)$ if $a$ is less than $f(q)$ and point $q$ destroys the image of $h$ in $H_{k}\left(F_{a}^{f(q)}\right)$. In Fig. $2\left[c_{0}\right]$ is a non-zero element in $H_{1}\left(f^{-1}\left(f\left(p_{0}\right)+\varepsilon\right)\right)$. It is also a non-zero element in $H_{1}\left(\underline{F}_{f\left(p_{0}\right)+\varepsilon}^{f\left(p_{7}\right)}\right)$ which is destroyed by $p_{7}$.

If point $q$ destroys $h \in H_{k}\left(f^{-1}(a)\right)$, then $H_{k}\left(F_{a^{\prime}}^{f(q)}\right) \rightarrow H_{k}\left(F_{a^{\prime}}^{f(q)} \cup\{q\}\right)$ is not an isomorphism for any $a^{\prime}$ where $a<a^{\prime}<f(q)$. (See Appendix B, Lemma 14.) Thus, if $q$ destroys $h \in H_{k}\left(f^{-1}(a)\right)$, then $q$ is an $H_{k}$-critical point for the interval sets of $f$.

A function $f: \Omega \rightarrow \mathbb{R}$ is point destructible if whenever $h \in H_{k}\left(F_{a}^{b}\right)$ is destroyed by $f^{-1}(b)$, then $h$ is destroyed by some point $q \in f^{-1}(b)$. Morse functions on smooth manifolds are point destructible. (See the next section for the definition of Morse functions.) Piecewise linear functions which have a different scalar value at each vertex of the underlying simplicial complex are also point destructible.

\section{5. $\quad$ Morse Functions}

Let $\Omega$ be a smooth, compact $d$-manifold and let $f: \Omega \rightarrow \mathbb{R}$ be a smooth map on $\Omega$. The critical points in Morse theory are the points $p$ such that the gradient of $f$ at $p$ is the zero vector. A critical point is non-degenerate if its Hessian has full rank. The index of a non-degenerate critical point is the number of negative eigenvalues of the Hessian. (See [11] or [10] for definition of the Hessian and further explanation of Morse theory.) Function $f$ is Morse if all its critical points are non-degenerate. If function $f$ is Morse, then its index $k$ critical points in Morse theory correspond to its $H_{k}$-critical points as defined in Section 2.3. 


\subsection{Interval Persistence}

Similar to the topological persistence, the interval persistence of a point $p \in \Omega$ measures the "age" of the "oldest" homology element destroyed by $p$. However, the homology elements are considered over interval sets as opposed to the sub-level sets. Formally, for each $k \geq 0$, the interval persistence of point $p \in \Omega$ is

$$
\Pi_{k}^{f}(p)=\sup \left\{f(p)-a: p \text { destroys some non-zero } h \in H_{k}\left(f^{-1}(a)\right)\right\} .
$$

We use sup in place of max because it is possible that $p$ destroys non-zero elements of $H_{k}\left(f^{-1}(a+\epsilon)\right)$ for any $\epsilon>0$ but not elements of $H_{k}\left(f^{-1}(a)\right)$. For example, in Fig. 2, $p_{7}$ destroys $\left[c_{0}\right]$ in $H_{1}\left(f^{-1}\left(f\left(p_{0}\right)+\epsilon\right)\right)$ but no element of $H_{1}\left(f^{-1}\left(f\left(p_{0}\right)\right)\right)$.

The persistent Betti numbers relate the homology groups of one space into the other. For $X \subseteq Y$ and $k \geq 0$, let $H_{k}^{X, Y}$ be the image of the map $H_{k}(X) \rightarrow H_{k}(Y)$ induced by inclusion $X \rightarrow Y$. Define

$$
\xi_{k}(X, Y)=\operatorname{dim} H_{k}^{X, Y}
$$

In words, $\xi_{k}(X, Y)$ counts the number of non-zero generators of $H_{k}(X)$ that remain so in the larger space $Y$. The persistent Betti numbers are defined as $\beta_{a}^{b}=\xi_{k}\left(F_{-\infty}^{a}, F_{-\infty}^{b}\right)$ for $a \leq b$ [4], [13].

Assume that $f$ has only a finite number of critical values for interval sets. Let $\delta$ be the minimum difference between any two critical values of $f$ and let $\epsilon$ equal $\delta / 2$. Define

$$
\mu_{a}^{b}=\left(\beta_{a+\epsilon}^{b-\epsilon}-\beta_{a+\epsilon}^{b+\epsilon}\right)-\left(\beta_{a-\epsilon}^{b-\epsilon}-\beta_{a-\epsilon}^{b+\epsilon}\right) .
$$

As noted in [4], $\mu_{a}^{b}$ counts the number of homology generators born at $a$ which die at $b$.

Edelsbrunner et al. [8] introduced a critical point pairing algorithm based on when a homology generator is born and killed. This algorithm is used in the context of Morse functions in [7]. It can be shown that a pair $(p, q)$ can be characterized by $\mu_{f(p)}^{f(q)}$. Specifically, two critical points $p$ and $q$ with $f(p)<f(q)$ are paired if and only if $\mu_{f(p)}^{f(q)}$ is positive. Edelsbrunner et al. [7], [8] define the topological persistence of $p$ and $q$ as $f(q)-f(p)$.

Interval persistence generalizes the notion of topological persistence in the following sense. One can show that a pair $(p, q)$ has a topological persistence $f(q)-f(p)$ only if it has an interval persistence $f(q)-f(p)$ though the converse is not necessarily true.

Theorem 2. Let $f$ be a Morse function where no two critical points share the same critical value. If a pair of critical points $(p, q)$ with $f(p)<f(q)$ has topological persistence $f(q)-f(p)$, then point $q$ has interval persistence $f(q)-f(p)$.

Proof of Theorem 2 is left to Appendix C. The converse of Theorem 2 is not true. Critical point $p_{7}$ in Fig. 2 destroys $\left[c_{0}\right]$ in $H_{1}\left(F_{f\left(p_{0}\right)}^{f\left(p_{7}\right)}\right)$. Thus $p_{7}$ has interval persistence $\Pi_{1}^{f}\left(p_{7}\right)=f\left(p_{7}\right)-f\left(p_{0}\right)$. Critical point $p_{6}$ in Fig. 2 joins the two connected components in $F_{f\left(p_{5}\right)}^{f\left(p_{6}\right)}$, destroying a homology element in $H_{0}\left(F_{f\left(p_{5}\right)}^{f\left(p_{6}\right)}\right)$. Thus $p_{6}$ has interval persistence $\Pi_{0}^{f}\left(p_{6}\right)=f\left(p_{6}\right)-f\left(p_{5}\right)$. On the other hand, for sub-level sets, points $p_{6}$ and $p_{7}$ create homology elements in $H_{1}\left(F_{-\infty}^{f\left(p_{6}\right)} \cup\left\{p_{6}\right\}\right)$ and $H_{2}\left(F_{-\infty}^{f\left(p_{7}\right)} \cup\left\{p_{7}\right\}\right)$, respectively, and 
these homology elements are never destroyed. Thus, points $p_{6}$ and $p_{7}$ have infinite (or undefined) topological persistence.

\section{Maps and Spaces}

We will be dealing with continuous functions on a compact, connected topological space, $\Omega$. We need some conditions that these functions will be well-behaved, i.e., have properties similar to Morse functions. However, we do not want to restrict ourselves to differentiable functions or to Morse functions.

For a function $f: \Omega \rightarrow \mathbb{R}$ and $a \in \mathbb{R}$, define the open $\epsilon$-neighborhood of $f^{-1}(a)$ as

$$
N_{\epsilon}\left(f^{-1}(a)\right)=\{x \in \Omega: a-\epsilon<f(x)<a+\epsilon\} .
$$

The first property we require is that the topology of $f^{-1}(a)$ is similar to the topology of an $\epsilon$-neighborhood of $f^{-1}(a)$ for suitably small $\epsilon$. The second property is that $f$ is point destructible. These properties are similar to the Morse condition that critical points are isolated. We define the first property more formally below.

Represent the unit interval $[0,1]$ by $I$. Subspace $X \subseteq Y$ is a strong deformation retract of $Y$ if there is a continuous $\varphi: Y \times I \rightarrow Y$ such that $\varphi(y, 0)=y$ and $\varphi(y, 1) \in X$ for all $y \in Y$ and $\varphi(x, t)=x$ for all $x \in X$ and $t \in[0,1]$. In other words, $\varphi$ continuously deforms $Y$ into $X$ without moving any points in $X$. If $X$ is a strong deformation retract of $Y$, then $H_{k}(X)$ is isomorphic to $H_{k}(Y)$.

Definition 5. The continuous function $f: \Omega \rightarrow \mathbb{R}$ is $L R$ (locally retractible) if, for all $a \in \mathbb{R}$, there exists some $\epsilon_{0}>0$ such that, for all $\epsilon \leq \epsilon_{0}$, the set $f^{-1}(a)$ is a strong deformation retract of $N_{\epsilon}\left(f^{-1}(a)\right)$.

Piecewise linear functions on compact spaces are LR. (See Appendix D, Lemma 18.) If a continuous function is Morse, then the function is LR. (See pp. 12-20 of [11] for a proof. Milnor actually proves that $\{x: f(x) \leq a\}$ is a deformation retract of $\{x: f(x) \leq a+\epsilon\}$ but his proof also shows that $f^{-1}(a)$ is a strong deformation retract of $\{x: a-\epsilon<f(x)<a+\epsilon\}$.) We use the following property of LR functions. (See Appendix $\mathrm{C}$ for proof.)

Lemma 2. Let $f: \Omega \rightarrow \mathbb{R}$ be a continuous, point destructible LR function. For any non-zero $h \in H_{k}\left(f^{-1}(a)\right)$, if $\underline{F}_{a}^{\infty}$ destroys $h$, then some point $q \in \Omega$ destroys $h$.

If point $q$ destroys some element of $H_{k}\left(f^{-1}\left(a_{1}\right)\right)$ and $a_{1}<a_{2}<f(q)$, does $q$ destroy some element of $H_{k}\left(f^{-1}\left(a_{2}\right)\right)$ ? The answer is yes.

Lemma 3. Let $f: \Omega \rightarrow \mathbb{R}$ be a continuous LR function. If $q \in \Omega$ destroys some non-zero $h_{1} \in H_{k}\left(f^{-1}\left(a_{1}\right)\right)$, then, for every $a_{2}$ where $a_{1}<a_{2}<f(q)$, there exists some non-zero $h_{2} \in H_{k}\left(f^{-1}\left(a_{2}\right)\right)$ such that point $q$ destroys $h_{2}$ and elements $h_{1}$ and $h_{2}$ have the same image $h$ in $H_{k}\left(\underline{F}_{a_{1}}^{f(q)}\right)$. 


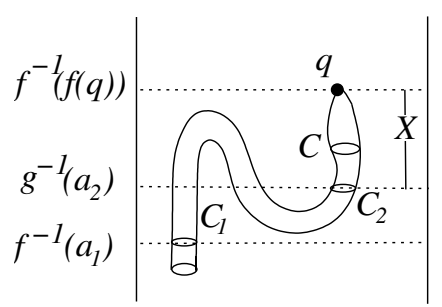

Fig. 4. Cycle $C$ generates $h \in H_{1}\left(\underline{F}_{a_{1}}^{f(q)}\right)$. Cycles $C_{1}$ and $C_{2}$ generate $h_{1} \in H_{1}\left(f^{-1}\left(a_{1}\right)\right)$ and $h_{2} \in$ $H_{1}\left(g^{-1}\left(a_{2}\right)\right)$, respectively, which are destroyed by $q$. Element $h$ is the image of both $h_{1}$ and $h_{2}$ in $H_{1}\left(F_{a_{1}}^{f(q)}\right)$.

The essence of the lemma is that if $h$ is the image of $h_{1} \in H_{k}\left(f^{-1}\left(a_{1}\right)\right)$ in $H_{k}\left(\underline{F}_{a_{1}}^{f(q)}\right)$ and $q$ destroys $h$, then some cycle in $f^{-1}\left(a_{2}\right)$ also generates $h$ in $H_{k}\left(\underline{F}_{a_{1}}^{f(q)}\right)$. The homology element in $H_{k}\left(f^{-1}\left(a_{2}\right)\right)$ generated by this cycle is also destroyed by $q$.

We also need a version of Lemma 3 which combines two functions (see Fig. 4).

Lemma 4. Let $f: \Omega \rightarrow \mathbb{R}$ and $g: \Omega \rightarrow \mathbb{R}$ be continuous LR functions and let $X=\left\{x \in \Omega: a_{2} \leq g(x)\right.$ and $\left.f(x)<f(q)\right\}$ for some $a_{2} \in \mathbb{R}$ and point $q \in \Omega$. If $q \in \Omega$ destroys some non-zero $h_{1} \in H_{k}\left(f^{-1}\left(a_{1}\right)\right)$ and $g^{-1}\left(a_{2}\right) \subseteq F_{a_{1}}^{f(q)}$, then there exists some non-zero $h_{2} \in H_{k}\left(g^{-1}\left(a_{2}\right)\right)$ such that point $q$ destroys the image of $h_{2}$ in $H_{k}(X)$ and elements $h_{1}$ and $h_{2}$ have the same image $h$ in $H_{k}\left(\underline{F}_{a_{1}}^{f(q)}\right)$.

Proofs of Lemmas 3 and 4 are left to Appendix D.

\section{Stability}

In this section we prove one of our main results, Theorem 3. Let $f$ and $g$ be two functions defined on $\Omega$. We say $|f-g|<\delta$ if $|f(x)-g(x)|<\delta$ for all $x \in \Omega$. Let $\gamma$ be some value greater than $2 \delta$. We show that if $q$ is $H_{k}$-critical for $f$ with interval persistence greater than or equal to $\gamma$, then there is an $H_{k}$-critical point $q^{\prime}$ for $g$ in the $(\gamma, 2 \delta)$-neighborhood of $q$ with interval persistence greater than $\gamma-2 \delta$. Moreover, the values $f(q)$ and $g\left(q^{\prime}\right)$ are close. This theorem not only relates $q$ and $q^{\prime}$ in the range as in [4] but also in the domain.

Consider Fig. 5 where $f$ is a function defined on the surface in $\mathbb{R}^{3}$ shown in the figure and $f(x)$ is the $z$-coordinate of the point $x$. The set $\{x \in \Omega: f(x) \leq f(r)\}$ is homeomorphic to a pinched cylinder with circles $c_{1}$ and $c_{2}$ bounding each end. The two maxima $p$ and $q$ destroy the elements $\left[c_{1}\right]$ and $\left[c_{2}\right]$ respectively in $H_{1}\left(f^{-1}(f(r))\right)$. We have $\Pi_{1}^{f}(p)=f(p)-f(r)$ and $\Pi_{1}^{f}(q)=f(q)-f(r)$. Now consider a slightly perturbed $f$ denoted as $g$. Set $g$ equal to $f$ everywhere except in the vicinity of $p$ and $q$ so that $|f-g|$ is much smaller than $\Pi_{1}^{f}(p)$ and $\Pi_{1}^{f}(q)$. Let $a$ be any value in between $f(p)$ and $f(r)$ where $f(p)-a>2|f-g|$. Our stability result asserts that there will be a maximum $p^{\prime}$ for $g$ where $p^{\prime} \in \underline{F}_{a}^{f(p)}$. Similar result holds for $q$ as well.

Theorem 3. Let $f, g: \Omega \rightarrow \mathbb{R}$ be continuous, point destructible LR functions on $\Omega$ where $|f-g|<\delta$. Further, let $q$ be an $H_{k}$-critical point that destroys a non-zero 


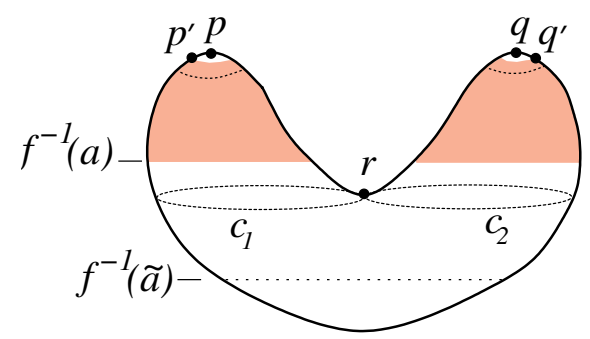

Fig. 5. Illustration that interval persistence cannot be replaced by topological persistence in Theorem 3 .

$h_{f} \in H_{k}\left(f^{-1}(a)\right)$. If $\gamma=f(q)-a>2 \delta$, then $\Pi_{k}^{f}(q)>\gamma$ and there is an $H_{k}$-critical point $q^{\prime}$ for $g$ in the $(\gamma, 2 \delta)$-neighborhood of $q$ such that

(i) $f(q)-\delta \leq g\left(q^{\prime}\right) \leq f(q)+\delta$, and

(ii) $\Pi_{k}^{g}\left(q^{\prime}\right)>\gamma-2 \delta$.

Topological persistence as in [4], [7], and [8] is based on sub-level sets. Trying to use topological persistence instead of interval persistence for matching critical points causes difficulties. Returning to Fig. 5, assume that $f(p)<f(q)$ whereas $g\left(p^{\prime}\right)>g\left(q^{\prime}\right)$. Points $p$ and $q^{\prime}$ are $H_{1}$-critical for interval sets. While $p^{\prime}$ is a critical point for sub-level sets of $g$, it is $H_{2}$-critical for sub-level sets, not $H_{1}$-critical, and it creates an $H_{2}$ homology element, instead of destroying one. The $H_{2}$ homology element created by $p^{\prime}$ is never destroyed and so $p^{\prime}$ is never matched and has infinite (or undefined) persistence.

Theorem 3 states that for every critical point $q$ for $f$ with persistence greater than $\gamma$, there is a critical point $q^{\prime}$ for $g$ in the $(\gamma, 2 \delta)$-neighborhood of $q$ with persistence greater than $\gamma-2 \delta$. The point $q^{\prime}$ depends upon the choice of $\gamma$. For instance, in Fig. 5, if $\gamma$ is set to $|f(p)-a|$, then point $p^{\prime}$ is in the $(\gamma, 2 \delta)$-neighborhood of $p$ and has persistence greater than $\gamma$. Similarly, point $q^{\prime}$ is in the $(\gamma, 2 \delta)$-neighborhood of $q$ and also has persistence greater than $\gamma$. If, instead of $\gamma$, we use $\tilde{\gamma}=|f(p)-\tilde{a}|$, then $p^{\prime}$ does not have persistence greater than $\tilde{\gamma}$ (nor does $q$.) Thus, point $p^{\prime}$ does not qualify for the role of $q^{\prime}$ in Theorem 3. Instead, $q^{\prime}$ is the point in the $(\tilde{\gamma}, 2 \delta)$-neighborhood of $p$ which has persistence greater than $\tilde{\gamma}$.

For the proof of Theorem 3, we need to restrict the homology element destroyed by some point $q$ to a connected component containing $q$. If $\tau$ is the connected component of $\underline{F}_{a}^{b} \cup\{q\}$ containing $q$ and $q$ destroys some non-zero element of $H_{k}\left(f^{-1}(a)\right)$, then $q$ destroys some non-zero element of $H_{k}\left(f^{-1}(a) \cap \tau\right)$. More generally, if $q$ destroys the non-zero image of some element of $H_{k}(\Omega)$ in $H_{k}\left(\underline{F}_{a}^{b} \cup\{q\}\right)$ for some $\Omega \subseteq \underline{F}_{a}^{b}$, then $q$ destroys the non-zero image of some element of $H_{k}(\Omega \cap \tau)$ in $H_{k}\left(\underline{F}_{a}^{b} \cup\{q\}\right)$. The statement of this lemma, Lemma 11, and its proof, are left to Appendix A.

Proof of Theorem 3. Let $b$ equal $f(q)$. Let

$$
X=\{x \in \Omega: a+\delta \leq g(x) \text { and } f(x)<b\} .
$$

Since $|f(x)-g(x)|<\delta$ and $b-a>2 \delta$, set $g^{-1}(a+\delta)$ is a subset of $F_{a}^{b}$. Note that 


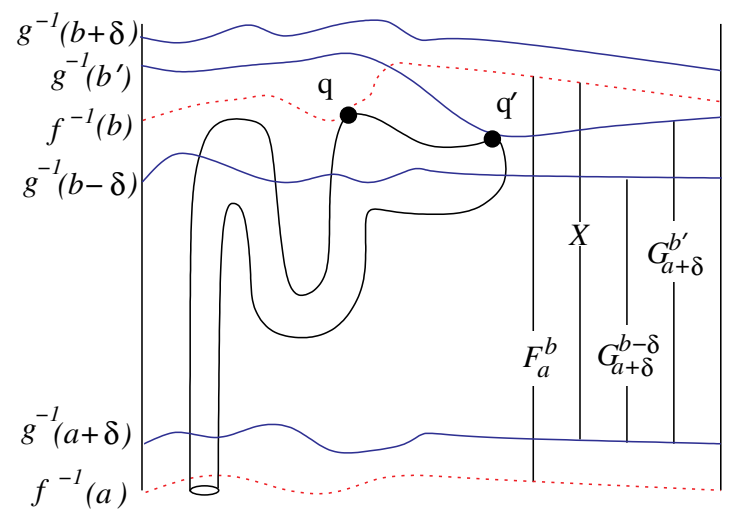

Fig. 6. Sets $\underline{F}_{a}^{b}, X=\{x \in \Omega: a+\delta \leq g(x)$ and $f(x)<b\}, \underline{G}_{a+\delta}^{b-\delta}$, and $\underline{G}_{a+\delta}^{b^{\prime}}$.

$\underline{G}_{a+\delta}^{b-\delta} \subseteq X \subseteq \underline{F}_{a}^{b}$ and so there exist homomorphisms $H_{k}\left(\underline{G}_{a+\delta}^{b-\delta}\right) \rightarrow H_{k}(X) \rightarrow H_{k}\left(F_{a}^{b}\right)$ induced by inclusions. (See Fig. 6.)

Let $h_{f}^{\prime} \in H_{k}\left(F_{a}^{b}\right)$ be the non-zero image of $h_{f}$ under the mapping $H_{k}\left(f^{-1}(a)\right) \rightarrow$ $H_{k}\left(\underline{F}_{a}^{b}\right)$. By Lemma 4, point $q$ destroys the image of some non-zero $\hat{h}_{g} \in H_{k}\left(g^{-1}(a+\delta)\right)$ where $h_{f}^{\prime}$ is the image of $\hat{h}_{g}$ under the mapping $H_{k}\left(g^{-1}(a+\delta)\right) \rightarrow H_{k}\left(F_{a}^{b}\right)$.

Let $\sigma^{f}$ be the pathwise connected component of $\underline{F}_{a}^{b} \cup\{q\}$ containing $q$. Let $\sigma$ equal $\sigma^{f} \cap g^{-1}(a+\delta)$. The mapping

$$
H_{k}\left(g^{-1}(a+\delta)\right) \rightarrow H_{k}\left(\underline{F}_{a}^{b}\right) \rightarrow H_{k}\left(\underline{F}_{a}^{b} \cup\{q\}\right)
$$

sends $\hat{h}_{g}$ to non-zero $h_{f}^{\prime} \in H_{k}\left(\underline{F}_{a}^{b}\right)$ to zero. By Lemma 11 in Appendix A, there is some non-zero $h_{\sigma} \in H_{k}(\sigma)$ such that the mapping

$$
H_{k}(\sigma) \rightarrow H_{k}\left(\underline{F}_{a}^{b}\right) \rightarrow H_{k}\left(\underline{F}_{a}^{b} \cup\{q\}\right)
$$

sends $h_{\sigma}$ to $h_{f}^{\prime}$ to zero.

Let $h_{g} \in H_{k}\left(g^{-1}(a+\delta)\right)$ be the image of $h_{\sigma}$ under the mapping $H_{k}(\sigma) \rightarrow H_{k}\left(g^{-1}(a+\right.$ $\delta)$ ). Let $h_{x}$ be the image of $h_{g}$ under the mapping $H_{k}\left(g^{-1}(a+\delta)\right) \rightarrow H_{k}(X)$. Since the mapping

$$
H_{k}(\sigma) \rightarrow H_{k}\left(g^{-1}(a+\delta)\right) \rightarrow H_{k}(X) \rightarrow H_{k}\left(\underline{F}_{a}^{b}\right)
$$

takes $h_{\sigma}$ to $h_{g}$ to $h_{x}$ to non-zero $h_{f}^{\prime}$, elements $h_{g}$ and $h_{x}$ must be non-zero. Since $h_{f}^{\prime}$ is destroyed by $q$, so is $h_{x}$. 
The following commutative diagram gives the relevant mappings between homology groups:

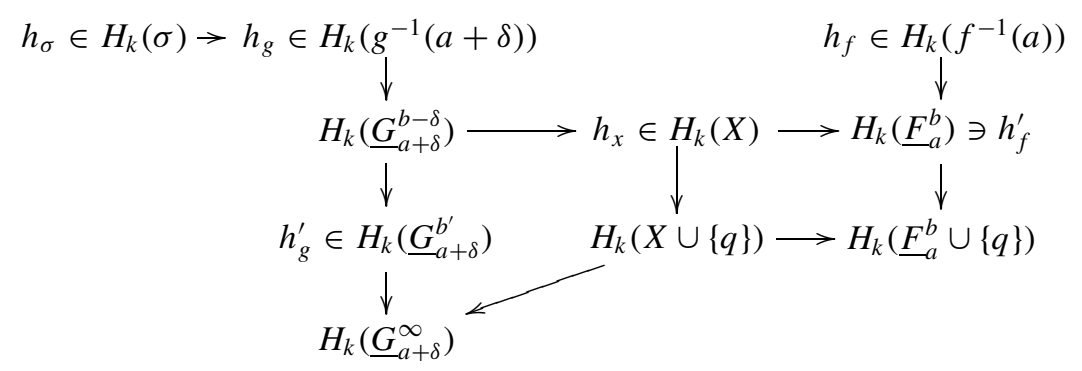

The value $b^{\prime}$ will be defined below.

Since $h_{x}$ is destroyed by $q$, the mapping $H_{k}(X) \rightarrow H_{k}(X \cup\{q\}) \rightarrow H_{k}\left(\underline{G}_{a+\delta}^{\infty}\right)$ sends $h_{x}$ to zero. Thus the composition of mappings $H_{k}\left(g^{-1}(a+\delta)\right) \rightarrow H_{k}(X) \rightarrow H_{k}\left(\underline{G}_{a+\delta}^{\infty}\right)$ sends $h_{g}$ to $h_{x}$ to zero.

By Lemma 2 there exists a point $q^{\prime} \in \Omega$ such that $h_{g}$ is destroyed by $q^{\prime}$ (i.e., the image of $h_{g}$ under the mapping $H_{k}\left(g^{-1}(a+\delta)\right) \rightarrow H_{k}\left(G_{a+\delta}^{g\left(q^{\prime}\right)}\right)$ is destroyed by $\left.q^{\prime}\right)$. Let $b^{\prime}$ equal $f\left(q^{\prime}\right)$. Since $\underline{G}_{a+\delta}^{b-\delta}$ is a subset of $X$, the image of $h_{g}$ under the mapping $H_{k}\left(g^{-1}(a+\delta)\right) \rightarrow H_{k}\left(\underline{G}_{a+\delta}^{b-\delta}\right)$ is non-zero and so

$$
b^{\prime} \geq b-\delta .
$$

Since $|f-g| \leq \delta$, set $X \cup f^{-1}(b)$ is a subset of $\underline{G}_{a+\delta}^{b+\delta} \cup g^{-1}(b+\delta)$ and so $h_{g}$ is destroyed by $\underline{G}_{a+\delta}^{b+\delta} \cup g^{-1}(b+\delta)$. Thus,

$$
b^{\prime} \leq b+\delta .
$$

Let $\sigma^{g}$ be the connected component of $\underline{G}_{a+\delta}^{b^{\prime}} \cup\left\{q^{\prime}\right\}$ containing $q^{\prime}$. We claim that $\sigma^{f}$ intersects $\sigma^{g}$.

The mapping

$$
H_{k}(\sigma) \rightarrow H_{k}\left(g^{-1}(a+\delta)\right) \rightarrow H_{k}\left(\underline{G}_{a+\delta}^{b^{\prime}}\right) \rightarrow H_{k}\left(\underline{G}_{a+\delta}^{b^{\prime}} \cup\left\{q^{\prime}\right\}\right)
$$

takes $h_{\sigma}$ to $h_{g}$ to some $h_{g}^{\prime} \in H_{k}\left(\underline{G}_{a+\delta}^{b^{\prime}}\right)$ to zero. Since point $q^{\prime}$ destroys $h_{g}$, element $h_{g}^{\prime}$ must be non-zero. By Lemma 11 there is some non-zero $h^{\prime \prime} \in H_{k}\left(\sigma \cap \sigma^{g}\right)$ such that $H_{k}\left(\sigma \cap \sigma^{g}\right) \rightarrow H_{k}\left(\underline{G}_{a+\delta}^{b^{\prime}}\right)$ takes $h^{\prime \prime}$ to $h_{g}^{\prime}$. Since $h^{\prime \prime}$ is non-zero, $H_{k}\left(\sigma \cap \sigma^{g}\right)$ is not the zero group and so $\sigma$ intersects $\sigma^{g}$. Since $\sigma$ is a subset of $\sigma^{f}$, set $\sigma^{f}$ also intersects $\sigma^{g}$.

Since $b^{\prime} \leq b+\delta$ and $|f-g|<\delta$, set $\underline{G}_{a+\delta}^{b^{\prime}} \cup\left\{q^{\prime}\right\}$ is contained in $\underline{F}_{a}^{b+2 \delta}$. Thus $\sigma^{g}$ is contained in $\underline{F}_{a}^{b+2 \delta}$.

Let $\hat{\sigma}$ be the connected component of $\underline{F}_{a}^{b+2 \delta}$ containing $q$. Its closure, $\operatorname{cl}(\hat{\sigma})$, is the $(\gamma, 2 \delta)$-neighborhood of $q$. Set $\hat{\sigma}$ contains $\sigma^{f}$. Since $\sigma^{g}$ intersects $\sigma^{f}$ and is connected, set $\hat{\sigma}$ also contains $\sigma^{g}$ and thus contains point $q^{\prime}$.

The claim $\Pi_{k}^{f}(q)>\gamma$ follows from definition since $f(q)-a=\gamma$. The point $q^{\prime}$ is $H_{k}$-critical since it destroys an element of $k$ th homology. It lies in $\operatorname{cl}(\hat{\sigma})$, the $(\gamma, 2 \delta)$ neighborhood of $q$. Proof of (i) follows from inequalities (4.1) and (4.2). Proof of (ii) follows from (i) and the fact that $q^{\prime}$ destroys $h_{g} \in H_{k}\left(g^{-1}(a+\delta)\right)$. 


\section{Computations}

\subsection{Computing Interval Persistence}

Theorem 3 can be used to compare two real-valued functions $f$ and $g$ defined on a topological space $\Omega$. The key computation to apply Theorem 3 is:

- determine if a point $p$ which destroys some $h \in H_{k}\left(f^{-1}(a)\right)$ has an interval persistence greater than a given value $\gamma$.

We use Betti numbers and their persistent counterparts to compute (i). We discuss the computations for the function $f$. It is clear that similar computations are needed for $g$ as well. In general, for a point $p$ and a value $a<b=f(p)$ we want to compute if an element of $H_{k}\left(f^{-1}(a)\right)$ gets destroyed by $p$. Let

$$
\begin{aligned}
\xi_{a}^{b} & =\xi_{k}\left(f^{-1}(a), \underline{F}_{a}^{b}\right) \quad \text { and } \\
\lambda_{a}^{p} & =\xi_{k}\left(f^{-1}(a), \underline{F}_{a}^{f(p)} \cup\{p\}\right) .
\end{aligned}
$$

Note that $p$ is a point whereas $a$ and $b$ are real values. The number $\xi_{a}^{b}$ counts the generators of $H_{k}\left(f^{-1}(a)\right)$ surviving in $F_{a}^{b}$ and $\lambda_{a}^{p}$ counts the generators of $H_{k}\left(f^{-1}(a)\right)$ surviving in $F_{a}^{b} \cup\{p\}$. Therefore,

$$
\pi_{a}^{p}=\xi_{a}^{b}-\lambda_{a}^{p}
$$

counts the generators of $H_{k}\left(f^{-1}(a)\right)$ destroyed by $p$. So, if $\pi_{a}^{p}>0$, we have an element of $H_{k}\left(f^{-1}(a)\right)$ that is destroyed by $p$ where $f(p)=b$.

Let $p_{0}, p_{1}, \ldots, p_{m}$ be the $H_{k}$-critical points of $f$ ordered according to the increasing values, that is, $f\left(p_{i}\right)>f\left(p_{i-1}\right)$ for all $i \geq 0$. We compute the interval persistence $\Pi_{k}^{f}\left(p_{j}\right)$ for these critical points $p_{i}$ as follows. For $1 \leq i \leq k-1$, let $a_{i}$ be a value with $f\left(p_{i-1}\right)<a_{i}<f\left(p_{i}\right)$. Compute $\pi_{a_{i}}^{p_{j}}$ for any pair $i, j$ where $j \geq i>0$. Since $\pi_{a}^{p_{j}}$ is constant for all $a$ where $f\left(p_{i-1}\right)<a<f\left(p_{i}\right)$, if $\pi_{a_{i}}^{p_{j}}$ is greater than zero, then the interval persistence $\Pi_{k}^{f}\left(p_{j}\right)$ is at least $f\left(p_{j}\right)-f\left(p_{i-1}\right)$. Thus we compute $\Pi_{k}^{f}\left(p_{j}\right)$ as

$$
\max _{i}\left|f\left(p_{j}\right)-f\left(p_{i-1}\right)\right| \text { so that } \pi_{a_{i}}^{p_{j}}>0 .
$$

Similarly, we can compute the critical points $q_{0}, q_{1}, \ldots, q_{n}$ and a set of intermittent values $b_{1}, b_{2}, \ldots, b_{n-1}$ for the function $g$. The interval persistence of an $H_{k}$-critical point $q$ of $g$ is measured similarly by $\Pi_{k}^{g}(q)$.

To compare $f$ and $g$, one can check, for each $k \geq 0$, if any critical point $p$ of $f$ has $\Pi_{k}^{f}(p)$ greater than a user-supplied parameter $\tau$. If so, search for a critical point $q$ of $g$ in the $(\tau, \tau)$-neighborhood of $q$ so that $\Pi_{k}^{g}(q)>\tau$ and $|f(p)-g(q)| \leq \tau / 2$. If $\tau>2 \delta$, such a $q$ exists by Theorem 3 .

\subsection{PL Case}

Assume there is some finite triangulation of $\Omega$ such that $f$ and $g$ are linear on each simplex of the triangulation. Functions $f$ and $g$ are LR (locally retractible), but not necessarily 
point destructible. A small perturbation of the scalar value at each triangulation vertex and the linear interpolation of those values over the triangulation simplices, gives new piecewise linear functions where no two vertices have the same scalar value. These new functions are point destructible and their vertices are located at the triangulation vertices.

Zomorodian and Carlsson in [13] show how to compute persistent Betti numbers for homology groups of filtered simplicial complexes over any field. However, spaces $\underline{F}_{a}^{b}$ and $F_{a}^{b} \cup\{p\}$ are not closed. To compute their persistent Betti numbers, $\xi_{a}^{b}$ and $\lambda_{a}^{p}$, we subdivide the simplicial complex and then extract a subcomplex with the same homology groups as $\underline{F}_{a}^{b}$ or $\underline{F}_{a}^{b} \cup\{p\}$.

Consider the space $\underline{F}_{a}^{b}$. Let $t \subseteq \Omega$ be a simplex which intersects this space. If $t$ is contained in $F_{-\infty}^{b}$ or $t$ is contained in $\underline{F}_{a}^{\infty}$, then $t \cap F_{a}^{b}$ can be collapsed to the face of $t$ contained in $\underline{F}_{a}^{b}$. Thus if for every simplex $t \in \Omega$ either $t \subseteq F_{-\infty}^{b}$ or $t \subseteq \underline{F}_{a}^{\infty}$, then $\underline{F}_{a}^{b}$ can be collapsed to the subcomplex $\Upsilon=\left\{t \in \Omega: t \subseteq \underline{F}_{a}^{b}\right\}$. However, there may be some simplices $t \in \Omega$ for which $t \cap F_{-\infty}^{a} \neq \emptyset$ and $t \cap \underline{F}_{b}^{\infty} \neq \emptyset$. We subdivide $\Omega$ to eliminate such simplices.

For each edge $e \in \Omega$, where $e \cap F_{-\infty}^{a} \neq \emptyset$ and $e \cap \underline{F}_{b}^{\infty} \neq \emptyset$, add a vertex $v_{e}$ in $e \cap \underline{F}_{a}^{b}$. Subdivide all the simplices $t$ containing $e$ by starring from $v_{e}$ to all the vertices of $t$. Repeat this until every edge of the complex is either a subset of $F_{-\infty}^{b}$ or of $\underline{F}_{a}^{\infty}$. Let $\tilde{\Omega}$ be the resulting subdivision of $\Omega$. Every edge in $\tilde{\Omega}$ is either a subset of $F_{-\infty}^{b}$ or of $\underline{F}_{a}^{\infty}$. (See Fig. 7.)

We claim that for every simplex $t \in \tilde{\Omega}$, either $t \subseteq F_{-\infty}^{b}$ or $t \subseteq \underline{F}_{a}^{\infty}$. If not, then $t$ has some vertex $v \in F_{-\infty}^{a}$ and some vertex $v^{\prime} \in \underline{F}_{b}^{\infty}$. Edge $\left(v, v^{\prime}\right) \in \tilde{\Omega}$ intersects both

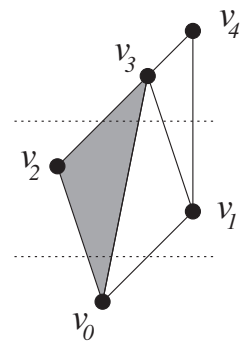

(a)

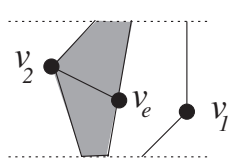

(c)

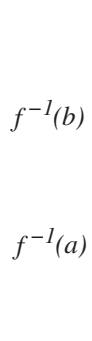

$f^{-1}(b)$

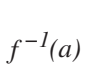

$f^{-1}(a)$

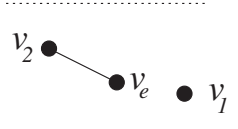

(d)

Fig. 7. (a) Simplicial complex $\Omega$ with edges $\left(v_{0}, v_{1}\right),\left(v_{0}, v_{2}\right),\left(v_{0}, v_{3}\right),\left(v_{1}, v_{3}\right),\left(v_{1}, v_{4}\right),\left(v_{2}, v_{3}\right),\left(v_{3}, v_{4}\right)$, and triangle $\left(v_{0}, v_{2}, v_{3}\right)$. For each point $(x, y)$ in the simplicial complex, $f(x, y)$ equals $y$. Edge $\left(v_{0}, v_{3}\right)$ intersects both $F_{-\infty}^{a}$ and $\underline{F}_{b}^{\infty}$. (b) Subdivision of edge $\left(v_{0}, v_{3}\right)$ and triangle $\left(v_{0}, v_{2}, v_{3}\right)$ by starring from $v_{e}$. (c) Set $\underline{F}_{a}^{b}$. (d) Deformation retract of $\underline{F}_{a}^{b}$ onto subcomplex $\Upsilon$. 
$F_{-\infty}^{a}$ and $\underline{F}_{b}^{\infty}$, a contradiction. Set $\Upsilon=\left\{t \in \tilde{\Omega}: t \subseteq \underline{F}_{a}^{b}\right\}$ is a deformation retract of $\underline{F}_{a}^{b}$ and therefore has homology groups isomorphic to that of $\underline{F}_{a}^{b}$. Apply the algorithm from [13] to compute the persistent Betti number $\beta_{a}^{b}$ of $\Upsilon$. The persistent Betti number $\beta_{a}^{b}$ of $\Upsilon$ equals $\xi_{a}^{b}$, the number of generators in $H_{k}\left(f^{-1}(a)\right)$ which were destroyed in $\underline{F}_{a}^{b}$. A similar algorithm can be used to compute $\lambda_{a}^{b}$.

\section{Maxima}

In this section we establish a stronger result for critical points that are maxima on oriented manifolds. We show that the $(\gamma, \infty)$-neighborhoods of local maxima with interval persistence greater than $\gamma$ are pairwise disjoint. This enables us to establish a matching of such critical points. Observe that Theorem 3 does not imply the disjointness of neighborhoods.

The idea of the proof is as follows. Consider two local maxima, $p_{0}, p_{1} \in \Omega$, with interval persistence greater than $\gamma$. Let $a_{0}$ equal $f\left(p_{0}\right)-\gamma$ and $a_{1}$ equal $f\left(p_{1}\right)-\gamma$. Let $\sigma_{0}$ and $\sigma_{1}$ be the $(\gamma, \infty)$-neighborhoods of $p_{0}$ and $p_{1}$, respectively. Set $\sigma_{i}$ is a connected component of $\underline{F}_{a_{i}}^{\infty}$. Without loss of generality, assume that $f\left(p_{0}\right)$ is less than or equal to $f\left(p_{1}\right)$.

Assume that $\sigma_{0}$ intersects $\sigma_{1}$. Since $\sigma_{0}$ intersects $\sigma_{1}$ and $a_{0} \leq a_{1}$, set $\sigma_{1}$ is contained in $\sigma_{0}$. Therefore, $p_{1}$ is contained in $\sigma_{0}$.

Since $p_{0}$ has persistence greater than $\gamma$, point $p_{0}$ destroys some non-zero $h \in H_{k}\left(\partial \sigma_{0}\right)$. Since $p_{0}$ is a local maximum, $k$ equals $d-1$. Under appropriate assumptions, $\sigma_{0}$ is a manifold with boundary. We then show that $\underline{F}_{a_{0}}^{f\left(p_{0}\right)} \cup\left\{p_{0}\right\}$ contains $\sigma_{0}$. Since $f\left(p_{0}\right) \leq$ $f\left(p_{1}\right)$, set $\underline{F}_{a_{0}}^{f\left(p_{0}\right)} \cup\left\{p_{0}\right\}$ does not contain $p_{1}$ and therefore point $p_{1}$ is not in $\sigma_{0}$, a contradiction. We conclude that $\sigma_{0}$ does not intersect $\sigma_{1}$.

We start the formal proof with a lemma about the homology groups destroyed by local maxima of $d$-manifolds. Lemmas 5 and 6 depend on some lemmas from homological algebra whose proofs are left to Appendices A and E.

Lemma 5. Let $\underline{F}_{a}^{\infty}$ be an oriented d-manifold with boundary and let $\sigma$ be the connected component of $\underline{F}_{a}^{\infty}$ containing $p$. If $p$ is a local maximum and p destroys $h \in H_{k}\left(f^{-1}(a)\right)$, then $k$ equals $d-1$ and $p$ destroys the image of some $h^{\prime} \in H_{d-1}(\partial \sigma)$ in $H_{d-1}\left(\underline{F}_{a}^{f(p)}\right)$.

Proof. Part I: Show that $k$ equals $d-1$.

Since $F_{a}^{\infty}$ is an oriented $d$-manifold and $p$ is a local maximum, some neighborhood $N_{p}$ of $p$ is homeomorphic to $\mathbb{R}^{d}$ and all points in $N_{p}-\{p\}$ have value less than $f(p)$. Let $B$ be the unit ball in $\mathbb{R}^{d}$, and let $B_{p}$ be its image under the homeomorphism from $\mathbb{R}^{d}$ to $N_{p}$. Since all points in $N_{p}-\{p\}$ have value less than $f(p)$, they are all in $\underline{F}_{a}^{f(p)}$.

Let $h^{\prime} \in H_{k}\left(\underline{F}_{a}^{f(p)}\right)$ be the non-zero image of $h \in H_{k}\left(f^{-1}(a)\right)$ under the mapping $H_{k}\left(f^{-1}(a)\right) \rightarrow H_{k}\left(\underline{F}_{a}^{f(p)}\right)$. The mapping $H_{k}\left(\underline{F}_{a}^{f(p)}\right) \rightarrow H_{k}\left(\underline{F}_{a}^{f(p)} \cup\{p\}\right)$ sends $h^{\prime}$ to zero. The intersection of $\underline{F}_{a}^{f(p)}$ and $B_{p}$ is $B_{p}-\{p\}$ and their union is $\underline{F}_{a}^{f(p)} \cup\{p\}$. By Corollary 8 (Appendix A), $h^{\prime}$ is the image of some $h^{\prime \prime} \in H_{k}\left(B_{p}-\{p\}\right)$ under the mapping $H_{k}\left(B_{p}-\{p\}\right) \rightarrow H_{k}\left(\underline{F}_{a}^{f(p)}\right)$. Since $h^{\prime}$ is non-zero, $h^{\prime \prime}$ is non-zero. Since $H_{k}\left(B_{p}-\{p\}\right)$ is the zero group, for all $k \neq d-1$, element $h^{\prime \prime}$ must be in $H_{d-1}\left(B_{p}-\{p\}\right)$. Therefore, 
$h$ and $h^{\prime}$ are elements of $H_{d-1}\left(f^{-1}(a)\right)$ and $H_{d-1}\left(F_{a}^{f(p)}\right)$, respectively, and so $k$ equals $d-1$.

Part II: Show that $p$ destroys the image of some $h^{\prime} \in H_{d-1}\left(f^{-1}(\partial \sigma)\right)$ in $H_{d-1}\left(F_{a}^{f(p)}\right)$. Let $\Omega$ equal $f^{-1}(a)$ and let $\Omega^{\prime}$ equal $\underline{F}_{a}^{f(p)} \cup\{p\}$. Let $\tau^{\prime}$ be the connected component of $\Omega^{\prime}$ containing $p$. Let $\tau$ equal $\tau^{\prime} \cap \Omega$. Note that $\tau$ equals $\partial \sigma$.

The mapping $H_{d-1}(\Omega) \rightarrow H_{d-1}\left(\Omega^{\prime}\right)$ sends $h \in H_{d-1}(\Omega)$ to zero while the mapping $H_{d-1}(\Omega) \rightarrow H_{d-1}\left(\Omega^{\prime}-\{p\}\right)$ does not. By Lemma 11 (Appendix A), the mapping $H_{d-1}(\tau) \rightarrow H_{d-1}\left(\Omega^{\prime}\right)$ sends some non-zero $h^{\prime} \in H_{d-1}(\tau)$ to zero while the mapping $H_{d-1}(\tau) \rightarrow H_{d-1}\left(\Omega^{\prime}-\{p\}\right)$ does not. Thus $p$ destroys the image of some $h^{\prime} \in H_{d-1}(\partial \sigma)=H_{d-1}(\tau)$ in $H_{d-1}\left(\underline{F}_{a}^{f(p)}\right)$.

Let $M$ be a connected, oriented $d$-manifold with non-empty boundary. We show that elements of $H_{d-1}(\partial M)$ are killed only by $M$, not by any subset of $M$.

Lemma 6. Let $M$ be a connected, oriented d-manifold with non-empty boundary. If $\partial M \subseteq M^{\prime} \subseteq M$ and $H_{d-1}(\partial M) \rightarrow H_{d-1}\left(M^{\prime}\right)$ takes some non-zero $h \in H_{d-1}(\partial M)$ to zero, then $M^{\prime}$ equals $M$.

Proof. Assume $M^{\prime}$ does not equal $M$. Let $p$ be a point in $M-M^{\prime}$. Let $B$ be an open topological ball containing $p$ whose closure does not intersect $\partial M$. There exists a deformation retract from $M-\{p\}$ to $M-B$. Thus $H_{d-1}(M-\{p\})$ is isomorphic to $H_{d-1}(M-B)$.

The mapping $H_{d-1}(\partial M) \rightarrow H_{d-1}\left(M^{\prime}\right) \rightarrow H_{d-1}(M-\{p\}) \rightarrow H_{d-1}(M-B)$ sends $h$ to zero in $H_{d-1}(M-B)$. Let $h_{0}$ be the element of the homology group of $H_{d-1}(\partial M)$ generated by $\partial M$ with orientation inherited from $M$. By Lemma 19 (Appendix E), element $h$ equals $\alpha h_{0}$ for some non-zero $\alpha$. Let $h_{B}$ be the element of $H_{d-1}(M-B)$ generated by $\partial B$ with orientation inherited from $M-B$. Let $h^{\prime}$ be the image of $h$ under the map $H_{d-1}(\partial M) \rightarrow H_{d-1}(\partial M \cup \partial B)$. The element $h$ and hence $h^{\prime}$ is sent to zero in $H_{d-1}(M-B)$. By Lemma 19 (Appendix E), element $h^{\prime}$ equals $\beta\left(h_{0}+h_{B}\right)$ for some non-zero $\beta$. Thus $\alpha h_{0}$ equals $\beta\left(h_{0}+h_{B}\right)$. Since $h_{0}$ and $h_{B}$ are linearly independent, $\alpha$ and $\beta$ are both zero implying $h$ is a zero element, a contradiction. It follows that $M$ equals $M^{\prime}$.

Let

$$
\sigma_{p}^{f}(\gamma)=(\gamma, \infty) \text { —neighborhood of } p \text { for } f .
$$

We prove that the neighborhoods $\sigma_{p}^{f}(\gamma)$ of maxima with interval persistence greater than $\gamma$ are pairwise disjoint.

Theorem 4. Let $f: \Omega \rightarrow \mathbb{R}$ be a continuous function such that $\underline{F}_{a}^{\infty}$ is a $(d-1)$ manifold with boundary for all but a finite number of a. If points $p_{0}, p_{1} \in \Omega$ are local maxima with interval persistence greater than $\gamma$, then $\sigma_{p_{0}}^{f}(\gamma)$ does not intersect $\sigma_{p_{1}}^{f}(\gamma)$.

Proof. Let $p_{0}, p_{1} \in \Omega$ be local maxima with persistence $\gamma_{0}, \gamma_{1}$, both greater than $\gamma$. Without loss of generality, assume that $f\left(p_{0}\right) \leq f\left(p_{1}\right)$. 


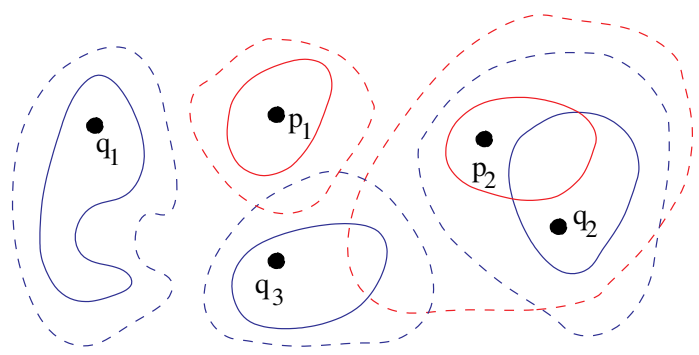

Fig. 8. Local maxima and their neighborhoods. Solid lines around points $p_{i}$ and $q_{i}$ are neighborhoods $\sigma_{p_{i}}^{f}(\gamma / 2)$ and $\sigma_{q_{i}}^{g}(\gamma / 2)$. Dotted lines are neighborhoods $\sigma_{p_{i}}^{f}(\gamma)$ and $\sigma_{q_{i}}^{g}(\gamma)$. Neighborhood $\sigma_{p_{2}}^{f}(\gamma / 2)$ intersects $\sigma_{q_{2}}^{g}(\gamma / 2)$ so $p_{2}$ matches with $q_{2}$. Point $p_{1}$ matches with some point (not shown) from $M_{g}(\gamma / 2)$ and points $q_{1}$ and $q_{3}$ match with points (not shown) from $M_{f}(\gamma / 2)$.

Assume that $\sigma_{p_{0}}^{f}(\gamma)$ intersects $\sigma_{p_{1}}^{f}(\gamma)$. Since $\underline{F}_{a}^{\infty}$ is a $(d-1)$-manifold for all but a finite number of $a$, there is some $\gamma^{\prime} \geq \gamma$ such that $\gamma_{0}>\gamma^{\prime}$ and $\gamma_{1}>\gamma^{\prime}$ and $\underline{F}_{f\left(p_{0}\right)-\gamma^{\prime}}^{\infty}$ is a $(d-1)$-manifold with boundary. Since $\sigma_{p_{0}}^{f}(\gamma)$ intersects $\sigma_{p_{1}}^{f}(\gamma)$, set $\sigma_{p_{0}}^{f}\left(\gamma^{\prime}\right)$ intersects $\sigma_{p_{1}}^{f}\left(\gamma^{\prime}\right)$.

Since $f\left(p_{0}\right) \leq f\left(p_{1}\right)$, set $\underline{F}_{f\left(p_{0}\right)-\gamma^{\prime}}^{\infty}$ contains $\underline{F}_{f\left(p_{1}\right)-\gamma^{\prime}}^{\infty}$. Since $\sigma_{p_{0}}^{f}\left(\gamma^{\prime}\right)$ intersects $\sigma_{p_{1}}^{f}\left(\gamma^{\prime}\right)$, set $\sigma_{p_{0}}^{f}\left(\gamma^{\prime}\right)$ contains $\sigma_{p_{1}}^{f}\left(\gamma^{\prime}\right)$. Thus $\sigma_{p_{0}}^{f}\left(\gamma^{\prime}\right)$ contains $p_{1}$.

By Lemma 3, point $p_{0}$ destroys some non-zero element of $H_{k}\left(f^{-1}\left(f\left(p_{0}\right)-\gamma^{\prime}\right)\right)$. By Lemma 5, $k$ equals $d-1$ and $p_{0}$ destroys a non-zero elements of $H_{d-1}\left(\partial \sigma_{p_{0}}^{f}\left(\gamma^{\prime}\right)\right)$. In Lemma 6 putting $M$ equal to $\sigma_{p_{0}}^{f}\left(\gamma^{\prime}\right)$ and $M^{\prime}$ equal to the connected component of $\underline{F}_{f\left(p_{0}\right)-\gamma^{\prime}}^{f\left(p_{0}\right)} \cup\left\{p_{0}\right\}$ containing $p_{0}$, we conclude $\underline{F}_{f\left(p_{0}\right)-\gamma^{\prime}}^{f\left(p_{0}\right)} \cup\left\{p_{0}\right\}$ equals $\sigma_{p_{0}}^{f}\left(\gamma^{\prime}\right)$ and thus contains $p_{1}$. However, since $f\left(p_{0}\right) \leq f\left(p_{1}\right)$, set $\underline{F}_{a}^{f\left(p_{0}\right)} \cup\left\{p_{0}\right\}$ does not contain $p_{1}$. Thus, $\sigma_{p_{0}}^{f}(\gamma)$ does not intersect $\sigma_{p_{1}}^{f}(\gamma)$.

Our final theorem gives relationships between neighborhoods of local maxima of $f$ and of $g$. See Fig. 8 where $\delta=\gamma / 4$.

Theorem 5. Let $f, g: \Omega \rightarrow \mathbb{R}$ be continuous functions such that $\underline{F}_{a}^{\infty}$ and $\underline{G}_{a}^{\infty}$ are $(d-1)$-manifolds with boundary for all but a finite number of $a$ and $|f-g|<\delta$. Let $p \in \Omega$ be a local maximum of $f$ and let $q$ and $q^{\prime}$ be local maxima of $g$ where $q \neq q^{\prime}$. Assume that $p, q, q^{\prime}$ have persistence greater than $\gamma$ and $|f(p)-g(q)|<\delta$ and $\left|f(p)-g\left(q^{\prime}\right)\right|<\delta$.

(i) If $\sigma_{p}^{f}(\gamma-2 \delta)$ intersects $\sigma_{q}^{g}(\gamma-2 \delta)$, then $\sigma_{q}^{g}(\gamma)$ contains $\sigma_{p}^{f}(\gamma-2 \delta)$.

(ii) If $\sigma_{p}^{f}(\gamma-2 \delta)$ intersects $\sigma_{q}^{g}(\gamma-2 \delta)$, then $\sigma_{p}^{f}(\gamma-2 \delta)$ does not intersect $\sigma_{q^{\prime}}^{g}(\gamma-2 \delta)$.

Proof of (i). Let $y$ be a point in $\sigma_{p}^{f}(\gamma-2 \delta) \cap \sigma_{q}^{g}(\gamma-2 \delta)$ and $z$ be any point in $\sigma_{p}^{f}(\gamma-2 \delta)$. Set $\sigma_{p}^{f}(\gamma-2 \delta)$ is path connected, so there is a path $\zeta \subseteq \sigma_{p}^{f}(\gamma-2 \delta)$ from $y$ to $z$. Since $\zeta \subseteq \sigma_{p}^{f}(\gamma-2 \delta), f(x) \geq f(p)-\gamma+2 \delta$ for every point $x \in \zeta$. Since $|f(x)-g(x)|<\delta$ for all $x \in \Omega$ and $|f(p)-g(q)|<\delta$, it follows that $g(x) \geq g(q)-\gamma$ 
for all $x \in \zeta$. Thus $\zeta \subseteq \sigma_{q}^{g}(\gamma)$ and $z$ lies in $\sigma_{q}^{g}(\gamma)$. This holds for all $z \in \sigma_{p}^{f}(\gamma-2 \delta)$ so $\sigma_{q}^{g}(\gamma)$ contains $\sigma_{p}^{f}(\gamma-2 \delta)$. (See the neighborhoods of $p_{2}$ and $q_{2}$ in Fig. 8.)

Proof of (ii). By Theorem 4, $\sigma_{q}^{g}(\gamma)$ and $\sigma_{q^{\prime}}^{g}(\gamma)$ are disjoint. By (i) above, if $\sigma_{p}^{f}(\gamma-2 \delta)$ intersects $\sigma_{q^{\prime}}^{g}(\gamma-2 \delta)$, then $\sigma_{q^{\prime}}^{g}(\gamma)$ contains $\sigma_{p}^{f}(\gamma-2 \delta)$. Then, by condition of (ii), $\sigma_{q^{\prime}}^{g}(\gamma)$ intersects $\sigma_{q}^{g}(\gamma-2 \delta)$ and hence $\sigma_{q}^{g}(\gamma)$. However, $\sigma_{q}^{g}(\gamma)$ and $\sigma_{q^{\prime}}^{g}(\gamma)$ are disjoint. Therefore, $\sigma_{p}^{f}(\gamma-2 \delta)$ cannot intersect $\sigma_{q^{\prime}}^{g}(\gamma-2 \delta$ ) (see Fig. 8).

\section{Matching}

We assume that $f, g: \Omega \rightarrow \mathbb{R}$ are continuous, point destructible LR functions such that $\underline{F}_{a}^{\infty}$ and $\underline{G}_{a}^{\infty}$ are $(d-1)$-manifolds with boundary for all but a finite number of $a$. Let $M_{f}$ and $M_{g}$ be the set of local maxima of $f$ and $g$, respectively, and let $M_{f}(\gamma) \subseteq M_{f}$ and $M_{g}(\gamma) \subseteq M_{g}$ be the set of local maxima of $f$ and $g$, respectively, with persistence greater than $\gamma$. We would like to match points in $M_{f}(\gamma)$ with close points in $M_{g}(\gamma)$ in the sense of Theorem 3. However, there may be no such matching. In fact, $f$ may contain a set of maxima with persistence a little bit above $\gamma$ while nearby critical points in $g$ all have persistence a bit below $\gamma$. Thus, $M_{f}(\gamma)$ can contain any number of points while $M_{g}(\gamma)$ is empty! Instead of matching $M_{f}(\gamma)$ and $M_{g}(\gamma)$ only with each other, we allow them to match with points with slightly less persistence.

We say that a partial matching of $M_{f}$ with $M_{g}$ covers $M_{f}(\gamma)$ and $M_{g}(\gamma)$ if all points in $M_{f}(\gamma)$ and $M_{g}(\gamma)$ are matched. A partial matching of $M_{f}$ and $M_{g}$ is $(\alpha, \beta)$-close if for each pair $(p, q)$ where $p \in M_{f}$ and $q \in M_{g}$, point $q$ lies in $\sigma_{p}^{f}(\alpha)$ and point $p$ lies in $\sigma_{q}^{g}(\alpha)$ and $|f(p)-g(q)|<\beta$.

Assume that $|f(x)-g(x)|<\gamma / 4$ for all $x \in \Omega$. We will find a partial matching of $M_{f}$ with $M_{g}$ which covers $M_{f}(\gamma)$ and $M_{g}(\gamma)$ and is $(\gamma, \gamma / 4)$-close.

The algorithm is as follows. For each point $p \in M_{f}(\gamma)$, we compute $\sigma_{p}=\sigma_{p}^{f}(\gamma / 2)$. Similarly, for each $q \in M_{g}(\gamma)$, we compute $\sigma_{q}=\sigma_{q}^{g}(\gamma / 2)$. By Theorem 5, each $\sigma_{p}$ intersects at most one $\sigma_{q}$ where $|f(p)-g(q)|<\gamma / 4$ and vice versa. If $\sigma_{p}$ intersects such a $\sigma_{q}$, then match $p$ with $q$. If not, then match $p$ with some $q^{\prime} \in M_{g}(\gamma / 2)$ lying in $\sigma_{p}$ such that $\left|f(p)-g\left(q^{\prime}\right)\right|<\gamma / 4$. (By Theorem 3 such a $q^{\prime}$ exists.) Similarly, if $\sigma_{q}$ does not intersect any $\sigma_{p}$, match $q$ with $p^{\prime} \in M_{f}(\gamma / 2)$ lying in $\sigma_{q}$ such that $\left|f\left(p^{\prime}\right)-g(q)\right|<\gamma / 4$.

We claim that algorithm MatchPersistentMax matches all maxima with persistence more than $\gamma$ :

Proposition 1. If $|f-g| \leq \gamma / 4$, then MATChPERSISTENTMAX $(\Omega, f, g, \gamma)$ produces a partial matching of $M_{f}$ with $M_{g}$ which is a subset of $M_{f}(\gamma / 2) \times M_{g}(\gamma / 2)$ and which covers $M_{f}(\gamma)$ and $M_{g}(\gamma)$ and which is $(\gamma, \gamma / 4)$-close.

Proof. By construction, $p \in M_{f}(\gamma / 2)$ and $q \in M_{g}(\gamma / 2)$ for every matched pair $(p, q)$. 
MAtChPERSistentMAX $(\Omega, f, g, \gamma)$

/* $f, g: \Omega \rightarrow \mathbb{R} * /$

1. Compute sets $M_{f}(\gamma)$ and $M_{g}(\gamma)$;

2. For each point $p \in M_{f}(\gamma)$, compute $\sigma_{p}=\sigma_{p}^{f}(\gamma / 2)$;

3. For each point $q \in M_{g}(\gamma)$, compute $\sigma_{q}=\sigma_{q}^{g}(\gamma / 2)$;

4. For each point $p \in M_{f}(\gamma)$ and $q \in M_{g}(\gamma)$, if $\sigma_{p} \cap \sigma_{q} \neq \emptyset$ and $|f(p)-g(q)|<$ $\gamma / 4$, then match $p$ with $q$;

5. For each unmatched $p \in M_{f}(\gamma)$, match $p$ with $q^{\prime} \in M_{g}(\gamma / 2) \cap \sigma_{p}$ where $\mid f(p)-$ $g\left(q^{\prime}\right) \mid<\gamma / 4$

6. For each unmatched $q \in M_{g}(\gamma)$, match $q$ with $p^{\prime} \in M_{f}(\gamma / 2) \cap \sigma_{q}$ where $\mid f\left(p^{\prime}\right)-$ $g(q) \mid<\gamma / 4$.

By Theorem 4 the $\sigma_{p}=\sigma_{p}^{f}(\gamma / 2), p \in M_{f}(\gamma)$, are pairwise disjoint and the $\sigma_{q}=$ $\sigma_{q}^{g}(\gamma / 2), q \in M_{g}(\gamma)$, are pairwise disjoint. By Theorem 5 each $\sigma_{p}$ intersects at most one $\sigma_{q}$ and vice versa. Thus Step 4 gives a one-to-one partial matching.

By Theorem $3, \sigma_{p}$ contains some point $q^{\prime} \in M_{g}(\gamma / 2)$ such that $\left|f(p)-g\left(q^{\prime}\right)\right| \leq \gamma / 4$. Since $\left(p, q^{\prime}\right)$ is not matched in Step 4, point $q^{\prime}$ is not in $M_{g}(\gamma)$. Thus point $q^{\prime}$ is not matched in Step 4. Since $\sigma_{p}$ does not intersect any $\sigma_{p^{\prime}}, p^{\prime} \in M_{f}(\gamma)$, point $q^{\prime}$ is matched to at most one $p$ in Step 5. Similarly, point $p^{\prime}$ in Step 6 is not matched in Steps 4 and 5 and is matched to at most one $q$ in Step 6 . Thus the matching is one to one and covers all of $M_{f}(\gamma)$ and $M_{g}(\gamma)$.

It remains to show that for each match $(p, q)$, set $\sigma_{p}^{f}(\gamma)$ contains $q$ and $\sigma_{q}^{g}(\gamma)$ contains $p$. If $p$ and $q$ are matched in Step 4, then $\sigma_{p}^{f}(\gamma / 2)$ intersects $\sigma_{q}^{g}(\gamma / 2)$. This holds true even if $p$ and $q$ are matched in Steps 5 or 6. By Theorem 5 with $\delta=\gamma / 4, \sigma_{p}^{f}(\gamma)$ contains $\sigma_{q}^{g}(\gamma / 2)$ which contains $q$ and $\sigma_{q}^{g}(\gamma)$ contains $\sigma_{p}^{f}(\gamma / 2)$ which contains $p$. Since points $p \in M_{f}$ and $q \in M_{g}$ are only matched if $|f(p)-g(q)|<\gamma / 4$, the matching is $(\gamma, \gamma / 4)$-close.

\section{Discussions}

Results on stability of topological persistence can be applied to shape distance functions. If we take a dense point sample from the boundary of a shape, the distance functions to the shape boundary and and its point sample are similar. Therefore, the results on topological persistence apply to the shape distance functions and their approximations by point samples. Previous works [2]-[4] have noted this application of topological persistence. It would be interesting to apply the results of this paper to these functions as well. Notably, our results in this paper have some connections to a shape matching algorithm proposed in [6]. According to our results, we can expect that distance functions of similar shapes have similar neighborhoods for maxima with large interval persistence. The algorithm in [6] uses maxima and their stable manifolds for matching. We suspect that these stable manifolds are playing the role of neighborhoods as suggested in this paper. Perhaps the performance of the matching algorithm in [6] can now be improved and better explained by our results. We plan to address this issue in future work. 
This research brings up some other interesting questions. We have obtained a stronger result for maxima than other critical points. Is it possible to extend this stronger result to other critical points? We have given an algorithm to compute interval persistence. How can this algorithm be made more efficient? Is there an efficient algorithm along the line of Cohen-Steiner et al. [5]? Theorem 2 tells us that for most of the critical points we can use the linear time algorithm recently discovered by Cohen-Steiner et al. [5] for topological persistence. It would be interesting to see how one may compute the interval persistence of the critical points that remain unpaired by topological persistence.

\section{Acknowledgement}

We thank Prof. Michael Davis from OSU Dept. of Mathematics for providing the proof of Lemma 19. We also thank Frédéric Chazal and David Cohen-Steiner for helpful comments on earlier versions of this paper.

\section{Appendix A. Homology}

A sequence of groups $G_{i}$ connected by homomorphisms form an exact sequence if any two consecutive homomorphisms in the sequence

$$
\cdots \rightarrow G_{i} \stackrel{\ell_{i}}{\rightarrow} G_{i+1} \stackrel{\ell_{i+1}}{\rightarrow} G_{i+2} \rightarrow \cdots
$$

satisfy the property that

$$
\operatorname{Im} \ell_{i}=\operatorname{Ker} \ell_{i+1}
$$

Let $A, B \subset X$ so that $X$ is the union of the interiors of $A$ and $B$ and $D=A \cap B$. The sequence

$$
H_{k}(D) \stackrel{\Phi}{\rightarrow} H_{k}(A) \oplus H_{k}(B) \stackrel{\Psi}{\rightarrow} H_{k}(X) \stackrel{\stackrel{\partial}{\rightarrow}}{\rightarrow} H_{k-1}(D)
$$

is exact and is called the Mayer-Vietoris sequence [9, p. 149]. The map $\partial$ is the connecting homomorphism given by boundary maps [9, p. 116].

We need the following lemmas and corollaries about exact sequences.

Lemma 7. Let

$$
H_{k}(A \cap B) \rightarrow H_{k}(A) \oplus H_{k}(B) \rightarrow H_{k}(A \cup B)
$$

be an exact sequence. If $H_{k}(A) \rightarrow H_{k}(A \cup B)$ maps $h_{a} \in H_{k}(A)$ to zero, then there exists some $h \in H_{k}(A \cap B)$ such that $H_{k}(A \cap B) \rightarrow H_{k}(A) \rightarrow H_{k}(A \cup B)$ maps $h$ to $h_{a}$ to zero.

Proof. The mapping $H_{k}(A) \oplus H_{k}(B) \rightarrow H_{k}(A \cup B)$ sends $h_{a} \oplus 0 \in H_{k}(A) \oplus H_{k}(B)$ to zero. Since

$$
H_{k}(A \cap B) \rightarrow H_{k}(A) \oplus H_{k}(B) \rightarrow H_{k}(A \cup B)
$$


is exact, $h_{a} \oplus 0$ is the image of some $h \in H_{k}(A \cap B)$ under the mapping $H_{k}(A \cap$ $B) \rightarrow H_{k}(A) \oplus H_{k}(B)$. Thus $h_{a}$ is the image of $h \in H_{k}(A \cap B)$ under the mapping $H_{k}(A \cap B) \rightarrow H_{k}(A)$.

We get the following corollary for subsets $A$ and $B$ of a topological space $X$ where $X$ is the union of the interiors of $A$ and $B$.

Corollary 8. Let $A$ and $B$ be subsets of a topological space $X$ where $X$ is the union of the interiors of $A$ and $B$. If $H_{k}(A) \rightarrow H_{k}(A \cup B)$ maps $h_{a} \in H_{k}(A)$ to zero, then there exists some $h \in H_{k}(A \cap B)$ such that $H_{k}(A \cap B) \rightarrow H_{k}(A) \rightarrow H_{k}(A \cup B)$ maps $h$ to $h_{a}$ to zero.

Proof. By Mayer-Vietoris, the sequence

$$
H_{k}(A \cap B) \rightarrow H_{k}(A) \oplus H_{k}(B) \rightarrow H_{k}(A \cup B)
$$

is exact. The result follows by Lemma 7.

Note that the interiors of $A$ and $B$ in Corollary 8 are taken with respect to $X$. Thus points on the boundary of $X$ may lie on the interior of $A$ or $B$.

Lemma 9. Let

$$
H_{k}(A \cap B) \rightarrow H_{k}(A) \oplus H_{k}(B) \rightarrow H_{k}(A \cup B)
$$

be an exact sequence. If $H_{k}(A) \rightarrow H_{k}(A \cup B)$ maps $h_{a} \in H_{k}(A)$ to $h^{\prime} \in H_{k}(A \cup B)$ and $H_{k}(B) \rightarrow H_{k}(A \cup B)$ maps $h_{b} \in H_{k}(B)$ to the same $h^{\prime} \in H_{k}(A \cup B)$ then there exists some $h \in H_{k}(A \cap B)$ such that $H_{k}(A \cap B) \rightarrow H_{k}(A) \rightarrow H_{k}(A \cup B)$ maps $h$ to $h_{a}$ to $h^{\prime}$ and $H_{k}(A \cap B) \rightarrow H_{k}(B) \rightarrow H_{k}(A \cup B)$ maps $h$ to $h_{b}$ to $h^{\prime}$.

Proof. The mapping $H_{k}(A) \oplus H_{k}(B) \rightarrow H_{k}(A \cup B)$ sends $\left(h_{a} \oplus h_{b}\right) \in H_{k}(A) \oplus H_{k}(B)$ to $\left(h^{\prime}-h^{\prime}\right)=0$. Since

$$
H_{k}(A \cap B) \rightarrow H_{k}(A) \oplus H_{k}(B) \rightarrow H_{k}(A \cup B)
$$

is exact, $\left(h_{a} \oplus h_{b}\right)$ is the image of some $h \in H_{k}(A \cap B)$ under the mapping $H_{k}(A \cap B) \rightarrow$ $H_{k}(A) \oplus H_{k}(B)$. Thus $h_{a}$ is the image of $h$ under the mapping $H_{k}(A \cap B) \rightarrow H_{k}(A)$ and $h_{b}$ is the image of $h$ under the mapping $H_{k}(A \cap B) \rightarrow H_{k}(B)$.

We have the following corollary for Lemma 9.

Corollary 10. Let $A$ and $B$ be subsets of a topological space $X$ where $X$ is the union of the interiors of $A$ and $B$. If $H_{k}(A) \rightarrow H_{k}(A \cup B)$ maps $h_{a} \in H_{k}(A)$ to $h^{\prime} \in H_{k}(A \cup B)$ and $H_{k}(B) \rightarrow H_{k}(A \cup B)$ maps $h_{b} \in H_{k}(B)$ to the same $h^{\prime} \in H_{k}(A \cup B)$ then there exists some $h \in H_{k}(A \cap B)$ such that $H_{k}(A \cap B) \rightarrow H_{k}(A) \rightarrow H_{k}(A \cup B)$ maps $h$ to $h_{a}$ to $h^{\prime}$ and $H_{k}(A \cap B) \rightarrow H_{k}(B) \rightarrow H_{k}(A \cup B)$ maps $h$ to $h_{b}$ to $h^{\prime}$. 
Proof. By Mayer-Vietoris, the sequence

$$
H_{k}(A \cap B) \rightarrow H_{k}(A) \oplus H_{k}(B) \rightarrow H_{k}(A \cup B)
$$

is exact. The result follows by Lemma 9 .

The following lemma restricts the homology element destroyed by some point $q$ to a connected component containing $q$. The lemma is used in the proof of Theorem 3 on the stability of critical points and in the proof of Lemma 5 on the local maxima of manifolds.

Lemma 11. Let $\Omega \subseteq \Omega^{\prime}$ be topological spaces, let $q$ be a point in $\Omega^{\prime}-\Omega$, let $\sigma^{\prime}$ be the pathwise connected component of $\Omega^{\prime}$ containing $q$ and let $\sigma$ equal $\sigma^{\prime} \cap \Omega$. If the mapping $H_{k}(\Omega) \rightarrow H_{k}\left(\Omega^{\prime}-\{q\}\right) \rightarrow H_{k}\left(\Omega^{\prime}\right)$, sends non-zero $h \in H(\Omega)$ to non-zero $h^{\prime} \in H_{k}\left(\Omega^{\prime}-\{q\}\right)$ to zero in $H_{k}\left(\Omega^{\prime}\right)$, then for some non-zero $h_{\sigma} \in H_{k}(\sigma)$ the mapping $H_{k}(\sigma) \rightarrow H_{k}\left(\Omega^{\prime}-\{q\}\right) \rightarrow H_{k}\left(\Omega^{\prime}\right)$ sends $h_{\sigma}$ to $h^{\prime}$ to zero.

Proof. Let $A$ equal $\Omega \cup\left(\Omega^{\prime}-\sigma^{\prime}\right)$ and let $B$ equal $\sigma^{\prime}$. Note that $A \cup B$ equals $\Omega^{\prime}$ while $A \cap B$ equals $\sigma$.

The mapping

$$
H(\Omega) \rightarrow H(A) \rightarrow H\left(\Omega^{\prime}-\{q\}\right) \rightarrow H\left(\Omega^{\prime}\right)
$$

takes $h$ to some $h_{a} \in H(A)$ to non-zero $h^{\prime} \in H\left(\Omega^{\prime}-\{q\}\right)$ to zero in $H\left(\Omega^{\prime}\right)$. Since $h^{\prime}$ is non-zero, element $h_{a}$ is also non-zero.

By Corollary 8, there is some $h_{\sigma} \in H_{k}(A \cap B)=H_{k}(\sigma)$ such that $H_{k}(\sigma) \rightarrow$ $H_{k}(A) \rightarrow H_{k}\left(\Omega^{\prime}\right)$ takes $h_{\sigma}$ to $h_{a}$ to zero. Since $H_{k}(A) \rightarrow H_{k}\left(\Omega^{\prime}-\{q\}\right) \rightarrow H_{k}\left(\Omega^{\prime}\right)$ sends $h_{a}$ to $h^{\prime}$ to zero, the mapping $H_{k}(\sigma) \rightarrow H_{k}\left(\Omega^{\prime}-\{q\}\right) \rightarrow H_{k}\left(\Omega^{\prime}\right)$ sends $h_{\sigma}$ to $h^{\prime}$ to zero. Since $h^{\prime}$ is non-zero, element $h_{\sigma}$ is non-zero.

\section{Appendix B. Critical Points}

Theorem 1 relates critical points for interval sets to critical points for sub-level sets. Whether a point is $H_{k}$-critical depends upon whether certain mappings of homology groups are isomorphisms. We present here the lemmas about isomorphisms of homology groups which are the basis of Theorem 1 . We also prove that if point $q$ destroys some element of $H_{k}$, then $f(q)$ is an $H_{k}$-critical value.

A short exact sequence is an exact sequence $0 \rightarrow H_{k}(X) \rightarrow H_{k}(Y) \rightarrow H_{k}(Z) \rightarrow 0$ where $H_{k}(X) \rightarrow H_{k}(Y)$ is an injection (one to one) and $H_{k}(Y) \rightarrow H_{k}(Z)$ is a surjection (onto) and the image of $H_{K}(X) \rightarrow H_{k}(Y)$ is the kernel of $H_{k}(Y) \rightarrow H_{k}(Z)$.

Lemma 12. Let

$$
0 \rightarrow H_{k}(A \cap B) \rightarrow H_{k}(A) \oplus H_{k}(B) \rightarrow H_{k}(A \cup B) \rightarrow 0
$$

be a short exact sequence of homology groups. Mapping $H_{k}(A \cap B) \rightarrow H_{k}(A)$ is an isomorphism if and only if $H_{k}(B) \rightarrow H_{k}(A \cup B)$ is an isomorphism. 
Proof. Part I: Assume that $H_{k}(A \cap B) \rightarrow H_{k}(A)$ is an isomorphism. Show that $H_{k}(B) \rightarrow H_{k}(A \cup B)$ is an isomorphism.

Assume that $H_{k}(B) \rightarrow H_{k}(A \cup B)$ was not injective. By definition, there exists a nonzero $h_{b} \in H_{k}(B)$ such that $H_{k}(B) \rightarrow H_{k}(A \cup B)$ maps $h_{b}$ to zero. Thus, the mapping $H_{k}(A) \oplus H_{k}(B) \rightarrow H_{k}(A \cup B)$ maps $0 \oplus h_{b}$ to zero. Since

$$
H_{k}(A \cap B) \rightarrow H_{k}(A) \oplus H_{k}(B) \rightarrow H_{k}(A \cup B)
$$

is exact, $0 \oplus h_{b}$ is the image of some $h \in H_{k}(A \cap B)$ under the mapping $H_{k}(A \cap B) \rightarrow$ $H_{k}(A) \oplus H_{k}(B)$. Since $H_{k}(A \cap B) \rightarrow H_{k}(A)$ is an isomorphism, element $h$ must equal zero. Since $h_{b}$ is the image of zero under the mapping $H_{k}(A \cap B) \rightarrow H_{k}(B)$, element $h_{b}$ must also equal zero, a contradiction. Thus $H_{k}(B) \rightarrow H_{k}(A \cup B)$ is injective.

Assume that $H_{k}(B) \rightarrow H_{k}(A \cup B)$ was not surjective. By definition, there exists a non-zero $h \in H_{k}(A \cup B)$ which is not in the image of $H_{k}(B) \rightarrow H_{k}(A \cup B)$. Since $H_{k}(A) \oplus H_{k}(B) \rightarrow H_{k}(A \cup B)$ is surjective, element $h$ is the image of some $h_{a} \oplus h_{b}$ under the mapping $H_{k}(A) \oplus H_{k}(B) \rightarrow H_{k}(A \cup B)$. Since $H_{k}(A \cap B) \rightarrow H_{k}(A)$ is an isomorphism, element $h_{a}$ is the image of some $h^{\prime} \in H_{k}(A \cap B)$ under the mapping $H_{k}(A \cap B) \rightarrow H_{k}(A)$. Let $h_{b}^{\prime}$ be the image of $h^{\prime}$ under the mapping $H_{k}(A \cap B) \rightarrow H_{k}(B)$. Since

$$
H_{k}(A \cap B) \rightarrow H_{k}(A) \oplus H_{k}(B) \rightarrow H_{k}(A \cup B)
$$

is exact, the mapping $H_{k}(A) \oplus H_{k}(B) \rightarrow H_{k}(A \cup B)$ sends $h_{a} \oplus h_{b}^{\prime}$ to zero. Since this mapping takes $h_{a} \oplus h_{b}$ to $h$ and $\left(h_{a} \oplus h_{b}\right)-\left(h_{a} \oplus h_{b}^{\prime}\right)$ equals $0 \oplus\left(h_{b}-h_{b}^{\prime}\right)$, this mapping takes $0 \oplus\left(h_{b}-h_{b}^{\prime}\right)$ to $h$. Element $h$ is the image of $h_{b}-h_{b}^{\prime}$, contradicting the assumption. Thus $H_{k}(B) \rightarrow H_{k}(A \cup B)$ is surjective.

Part II: Assume that $H_{k}(B) \rightarrow H_{k}(A \cup B)$ is an isomorphism. Show that $H_{k}(A \cap B) \rightarrow$ $H_{k}(A)$ is an isomorphism.

Assume that $H_{k}(A \cap B) \rightarrow H_{k}(A)$ is not injective. By definition, there exists an $h \in$ $H_{k}(A \cap B)$ such that $H_{k}(A \cap B) \rightarrow H_{k}(A)$ maps $h$ to zero. Since $H_{k}(A) \rightarrow H_{k}(A \cup B)$ sends zero to zero, the mapping $H_{k}(A \cap B) \rightarrow H_{k}(A \cup B)$ also maps $h$ to zero. The composition $H_{k}(A \cap B) \rightarrow H_{k}(A) \oplus H_{k}(B) \rightarrow H_{k}(A \cup B)$ maps $h$ to $0 \oplus h_{b}$ to zero for some $h_{b} \in H_{k}(B)$. Since $H_{k}(B) \rightarrow H_{k}(A \cup B)$ is an isomorphism, element $h_{b}$ equals zero. Thus the mapping $H_{k}(A \cap B) \rightarrow H_{k}(A) \oplus H_{k}(B)$ sends $h$ to $0 \oplus 0$, the zero element of $H_{k}(A) \oplus H_{k}(B)$. Since $H_{k}(A \cap B) \rightarrow H_{k}(A) \oplus H_{k}(B)$ is injective, element $h$ must be zero, a contradiction. Thus, $H_{k}(A \cap B) \rightarrow H_{k}(A)$ is injective.

Assume that $H_{k}(A \cap B) \rightarrow H_{k}(A)$ is not surjective. By definition, there exists an $h_{a} \in H_{k}(A)$ which is not in the image of $H_{k}(A \cap B) \rightarrow H_{k}(A)$. Thus, $h_{a} \oplus 0$ is not in the image of $H_{k}(A \cap B) \rightarrow H_{k}(A) \oplus H_{k}(B)$. The mapping $H_{k}(A) \oplus H_{k}(B) \rightarrow H_{k}(A \cup B)$ takes $h_{a} \oplus 0$ to some $h \in H_{k}(A \cup B)$. Since $h_{a} \oplus 0$ is not in the image of $H_{k}(A \cap B) \rightarrow$ $H_{k}(A) \oplus H_{k}(B)$ and

$$
H_{k}(A \cap B) \rightarrow H_{k}(A) \oplus H_{k}(B) \rightarrow H_{k}(A \cup B)
$$

is exact, element $h$ is not equal to zero. Since $H_{k}(B) \rightarrow H_{k}(A \cup B)$ is an isomorphism, there exists an $h_{b} \in H_{k}(B)$ such that $H_{k}(B) \rightarrow H_{k}(A \cup B)$ maps $h_{b}$ to $h$. The mapping $H_{k}(A) \oplus H_{k}(B) \rightarrow H_{k}(A \cup B)$ takes $h_{a} \oplus h_{b}$ to $h-h=0$. Thus, $h_{a} \oplus h_{b}$ is in the image of $H_{k}(A \cap B) \rightarrow H_{k}(A) \oplus H_{k}(B)$ and $h_{a}$ is in the image of $H_{k}(A \cap B) \rightarrow H_{k}(A)$, a contradiction. We conclude that $H_{k}(A \cap B) \rightarrow H_{k}(A)$ is surjective. 
Lemma 13. Let $A$ and $B$ be topological spaces such that

$$
\begin{aligned}
H_{k}(A \cap B) \rightarrow H_{k}(A) \oplus H_{k}(B) \rightarrow & H_{k}(A \cup B) \\
\downarrow & \\
& H_{k-1}(A \cap B)
\end{aligned}
$$

is exact for all $k \geq 0$. Mapping $H_{k}(A \cap B) \rightarrow H_{k}(A)$ is an isomorphism for all $k$ if and only if mapping $H_{k}(B) \rightarrow H_{k}(A \cup B)$ is an isomorphism for all $k$.

Proof. Part I: Assume that $H_{k}(A \cap B) \rightarrow H_{k}(A)$ is an isomorphism for all $k$. Show that $H_{k}(B) \rightarrow H_{k}(A \cup B)$ is an isomorphism for all $k$.

Since $H_{k}(A \cap B) \rightarrow H_{k}(A)$ is injective for all $k$, mapping $H_{k}(A \cap B) \rightarrow H_{k}(A) \oplus$ $H_{k}(B)$ is also injective for all $k$. Since

$$
H_{k}(A \cup B) \rightarrow H_{k-1}(A \cap B) \rightarrow H_{k-1}(A) \oplus H_{k-1}(B)
$$

is exact and $H_{k-1}(A \cap B) \rightarrow H_{k-1}(A) \oplus H_{k-1}(B)$ is injective, the image of $H_{k}(A \cup B) \rightarrow$ $H_{k-1}(A \cap B)$ is zero. Since

$$
H_{k}(A) \oplus H_{k}(B) \rightarrow H_{k}(A \cup B) \rightarrow H_{k-1}(A \cap B)
$$

is exact and the image of $H_{k}(A \cup B) \rightarrow H_{k-1}(A \cap B)$ is zero, $H_{k}(A \cup B)$ is the image of $H_{k}(A) \oplus H_{k}(B) \rightarrow H_{k}(A \cup B)$. Thus, $H_{k}(A) \oplus H_{k}(B) \rightarrow H_{k}(A \cup B)$ is surjective and

$$
0 \rightarrow H_{k}(A \cap B) \rightarrow H_{k}(A) \oplus H_{k}(B) \rightarrow H_{k}(A \cup B) \rightarrow 0
$$

forms a short exact sequence. By Lemma 12, if $H_{k}(A \cup B) \rightarrow H_{k}(A)$ is an isomorphism, then $H_{k}(B) \rightarrow H_{k}(A \cup B)$ is an isomorphism.

Part II: Assume that $H_{k}(B) \rightarrow H_{k}(A \cup B)$ is an isomorphism for all $k$. Show that $H_{k}(A \cap B) \rightarrow H_{k}(A)$ is an isomorphism for all $k$.

Since $H_{k}(B) \rightarrow H_{k}(A \cup B)$ is surjective for all $k$, the mapping $H_{k}(A) \oplus H_{k}(B) \rightarrow$ $H_{k}(A \cup B)$ is surjective for all $k$. Since

$$
H_{k+1}(A) \oplus H_{k+1}(B) \rightarrow H_{k+1}(A \cup B) \rightarrow H_{k}(A \cap B)
$$

is exact and $H_{k+1}(A) \oplus H_{k+1}(B) \rightarrow H_{k+1}(A \cup B)$ is surjective, the image of $H_{k+1}(A \cup$ $B) \rightarrow H_{k}(A \cap B)$ is zero. Since

$$
H_{k+1}(A \cup B) \rightarrow H_{k}(A \cap B) \rightarrow H_{k}(A) \oplus H_{k}(B)
$$

is exact and the image of $H_{k+1}(A \cup B) \rightarrow H_{k}(A \cap B)$ is zero, the mapping $H_{k}(A \cap B) \rightarrow$ $H_{k}(A) \oplus H_{k}(B)$ is injective and

$$
0 \rightarrow H_{k}(A \cap B) \rightarrow H_{k}(A) \oplus H_{k}(B) \rightarrow H_{k}(A \cup B) \rightarrow 0
$$

forms a short exact sequence. By Lemma 12, if $H_{k}(B) \rightarrow H_{k}(A \cup B)$ is an isomorphism. then $H_{k}(A \cup B) \rightarrow H_{k}(A)$ is an isomorphism. 
As a corollary, we get the relationship between critical points for interval sets and critical points for sub-level sets given by Lemma 1 .

Proof of Lemma 1. Let $A$ equal $F_{-\infty}^{f(p)}$ and $B$ equal $F_{a}^{f(p)} \cup\{p\}$. By Mayer-Vietoris,

$$
\begin{array}{r}
H_{k}\left(F_{a}^{f(p)}\right) \rightarrow H_{k}(A) \oplus H_{k}(B) \rightarrow H_{k}\left(F_{-\infty}^{f(p)} \cup\{p\}\right) \\
\downarrow \\
H_{k-1}\left(F_{a}^{f(p)}\right)
\end{array}
$$

is an exact sequence for all $k$. By Lemma $13, H_{k}\left(F_{a}^{f(p)}\right) \rightarrow H_{k}\left(F_{a}^{f(p)} \cup\{p\}\right)$ is an isomorphism for all integers $k \geq 0$ if and only if $H_{k}\left(F_{-\infty}^{f(p)}\right) \rightarrow H_{k}\left(F_{-\infty}^{f(p)} \cup\{p\}\right)$ is an isomorphism for all integers $k \geq 0$.

Finally, we prove that if point $q$ destroys some element of $H_{k}$, then $f(q)$ is an $H_{k^{-}}$ critical value.

Lemma 14. If point $q$ destroys some non-zero $h \in H_{k}\left(f^{-1}(a)\right)$, then $H_{k}\left(F_{a^{\prime}}^{f(q)}\right) \rightarrow$ $H_{k}\left(F_{a^{\prime}}^{f(q)} \cup\{q\}\right)$ is not an isomorphism for any $a^{\prime}$ where $a<a^{\prime}<f(q)$.

Proof. Let $A$ equal $\underline{F}_{a}^{f(q)}$ and $B$ equal $\underline{F}_{a^{\prime}}^{f(q)} \cup\{q\}$. Note that $A \cup B$ equals $\underline{F}_{a}^{f(q)} \cup\{q\}$ and $A \cap B$ equals $\underline{F}_{a^{\prime}}^{f(q)}$.

Let $h_{a} \in H_{k}(A)$ be the image of $h$ under the mapping $H_{k}\left(f^{-1}(a)\right) \rightarrow H_{k}(A)$. Since $q$ destroys $h$, element $h_{a}$ is non-zero and the mapping $H_{k}(A) \rightarrow H_{k}(A \cup B)$ sends $h_{a}$ to zero. By Corollary 8, there is some $h^{\prime} \in H_{k}(A \cap B)$ such that $H_{k}(A \cap B) \rightarrow H_{k}(A) \rightarrow$ $H_{k}(A \cup B)$ sends $h^{\prime}$ to $h_{a}$ to zero. Since $h_{a}$ is non-zero, element $h^{\prime}$ is non-zero. Thus the mapping $H_{k}(A \cap B) \rightarrow H_{k}(A \cup B)$ is not an isomorphism.

\section{Appendix C. Interval vs. Topological Persistence}

In this section we prove Theorem 2 showing that interval persistence generalizes topological persistence for Morse functions. Morse functions have the property that for all $a \in \mathbb{R}$, there exists some $\epsilon_{0}>0$ such that, for all $\epsilon \leq \epsilon_{0}$, the set $f^{-1}(a)$ is a strong deformation retract of $N_{\epsilon}\left(f^{-1}(a)\right)=\{x: a-\epsilon<f(x)<a+\epsilon\}$. Note that if $f^{-1}(a)$ is a strong deformation retract of $N_{\epsilon}\left(f^{-1}(a)\right)$, then $H_{k}\left(f^{-1}(a)\right) \rightarrow H_{k}\left(N_{\epsilon}\left(f^{-1}(a)\right)\right)$ is an isomorphism for every integer $k \geq 0$.

As in Section 2.3, let $\delta$ be the minimum distance between any two critical values of $f$ and let $\epsilon$ equal $\delta / 2$. As defined in Section 2.1, $\beta_{k}(X)$ represent the $k$ th Betti number of $X$, i.e., the dimension of $H_{k}(X)$.

Since we consider homology groups defined only over fields, the homology groups have no torsion. Thus, if $p$ is the only critical point of $f$ with critical value $b=f(p)$, then, for some integer $k, \beta_{k}\left(F_{-\infty}^{b+\epsilon}\right)$ equals $\beta_{k}\left(F_{-\infty}^{b-\epsilon}\right) \pm 1$ and $\beta_{j}\left(F_{-\infty}^{b+\epsilon}\right)$ equals $\beta_{j}\left(F_{-\infty}^{b-\epsilon}\right)$ for all $j \neq k$. The proof (omitted) follows from the theorem [10, Theorem 3.1] that there exists a $k$-cell, $\omega$, in $\Omega$, such that $\omega \cap F_{-\infty}^{b-\epsilon}=\partial \omega$, and there is a deformation retract from $F_{-\infty}^{b+\epsilon}$ onto $F_{-\infty}^{b-\epsilon} \cup \omega$. 
Since the homology groups have no torsion, if $\beta_{j}\left(F_{-\infty}^{b-\epsilon}\right)$ equals $\beta_{j}\left(F_{-\infty}^{b+\epsilon}\right)$ then $H_{j}\left(F_{-\infty}^{b-\epsilon}\right) \rightarrow H_{j}\left(F_{-\infty}^{b+\epsilon}\right)$ is an isomorphism. If $\beta_{j}\left(F_{-\infty}^{b-\epsilon}\right)$ equals $\beta_{j}\left(F_{-\infty}^{b+\epsilon}\right)+1$ and only a single non-degenerate critical point has critical value $b$, then $H_{j}\left(F_{-\infty}^{b-\epsilon}\right) \rightarrow H_{j}\left(F_{-\infty}^{b+\epsilon}\right)$ is a surjection. If $\beta_{j}\left(F_{-\infty}^{b-\epsilon}\right)$ equals $\beta_{j}\left(F_{-\infty}^{b+\epsilon}\right)-1$ and only a single non-degenerate critical point has critical value $b$, then $H_{j}\left(F_{-\infty}^{b-\epsilon}\right) \rightarrow H_{j}\left(F_{-\infty}^{b+\epsilon}\right)$ is an injection.

In Section 2.6 we defined $\mu_{a}^{b}$ as

$$
\left(\beta_{a+\epsilon}^{b-\epsilon}-\beta_{a+\epsilon}^{b+\epsilon}\right)-\left(\beta_{a-\epsilon}^{b-\epsilon}-\beta_{a-\epsilon}^{b+\epsilon}\right) .
$$

Two critical points $p$ and $q$ with $f(p)<f(q)$ are paired if and only if $\mu_{f(p)}^{f(q)}$ is positive. We call $(p, q)$ a pair of critical points and $(f(p), f(q))$ a pair of critical values.

For the proof of Theorem 2, we need a lemma which shows that if $(a, b)$ is a pair of critical values and no two critical points share the same critical values, then $\mu_{a}^{b}$ equals one and $\mu_{a^{\prime}}^{b}$ equals zero for all critical values $a^{\prime} \neq a$. Fix the homology group index $k$. For any $x, y \in \mathbb{R}$ where $x<y$, let $W_{x}^{y}$ be the image of $H_{k}\left(F_{-\infty}^{x}\right)$ under the mapping $H_{k}\left(F_{-\infty}^{x}\right) \rightarrow H_{k}\left(F_{-\infty}^{y}\right)$, i.e., $W_{x}^{y}$ is a persistent homology group. The persistent Betti number $\beta_{x}^{y}$ is the dimension of $W_{x}^{y}$.

Lemma 15. Let $f$ be a Morse function where no two critical points share the same critical value. If $(a, b)$ are a pair of critical values, then

1. $\mu_{a}^{b}$ equals one;

2. $\mu_{a^{\prime}}^{b}$ equals zero for all critical values $a^{\prime} \neq a$;

3. $\beta_{a^{\prime \prime}}^{b-\epsilon}-\beta_{a^{\prime \prime}}^{b+\epsilon}$ equals zero for all $a^{\prime \prime}<a$.

Proof. Let $x, x^{\prime}, y, y^{\prime}$ be reals such that $x^{\prime} \leq x<y \leq y^{\prime}$. We prove that $\beta_{x}^{y} \geq \beta_{x^{\prime}}^{y^{\prime}}$. Intuitively, any homology group which persists from $x^{\prime}$ to $y^{\prime}$ surely persists from $x$ to $y$. More formally, we define an injective mapping $\Phi$ which takes each element of $W_{x^{\prime}}^{y^{\prime}}$ to a distinct element of $W_{x}^{b}$.

Define the mapping $\Phi: W_{x^{\prime}}^{y^{\prime}} \rightarrow W_{x}^{y}$ as follows. Every $h_{y}^{\prime} \in W_{x^{\prime}}^{y^{\prime}} \subseteq H_{k}\left(F_{-\infty}^{y^{\prime}}\right)$ is the image of some $h_{x}^{\prime} \in H_{k}\left(F_{-\infty}^{x^{\prime}}\right)$ under the mapping $H_{k}\left(F_{-\infty}^{x^{\prime}}\right) \rightarrow H_{k}\left(F_{-\infty}^{y^{\prime}}\right)$. Let $h_{x}$ be the image of $h_{x}^{\prime}$ under the mapping $H_{k}\left(F_{-\infty}^{x^{\prime}}\right) \rightarrow H_{k}\left(F_{-\infty}^{x}\right)$ and let $h_{y}$ be the image of $h_{x}$ under the mapping $H_{k}\left(F_{-\infty}^{x}\right) \rightarrow H_{k}\left(F_{-\infty}^{y}\right)$. The mappings are

$$
h_{x}^{\prime} \in H_{k}\left(F_{-\infty}^{x^{\prime}}\right) \rightarrow h_{x} \in H_{k}\left(F_{-\infty}^{x}\right) \rightarrow h_{y} \in H_{k}\left(F_{-\infty}^{y}\right) \rightarrow h_{y}^{\prime} \in H_{k}\left(F_{-\infty}^{y^{\prime}}\right) .
$$

Define $\Phi\left(h_{y}^{\prime}\right)=h_{y}$. Note that $h_{y}^{\prime}$ is the image of $h_{y}$ under the mapping $H_{k}\left(F_{-\infty}^{y^{\prime}}\right) \rightarrow$ $H_{k}\left(F_{-\infty}^{y}\right)$, so if $h_{y}$ is zero, then $h_{y}^{\prime}$ is zero. Thus $\Phi$ is injective and $\beta_{x}^{y}$ is greater than or equal to $\beta_{x^{\prime}}^{y^{\prime}}$. In particular, $\beta_{a+\epsilon}^{b-\epsilon} \geq \beta_{a+\epsilon}^{b+\epsilon}$ and $\beta_{a-\epsilon}^{b-\epsilon} \geq \beta_{a-\epsilon}^{b+\epsilon}$.

Next we show that $\beta_{x^{\prime}}^{y}-\beta_{x^{\prime}}^{y^{\prime}} \leq \beta_{x}^{y}-\beta_{x}^{y^{\prime}}$. The difference $\beta_{x^{\prime}}^{y}-\beta_{x^{\prime}}^{y^{\prime}}$ represents the rank of the homology groups which exist at $x^{\prime}$ and are destroyed between $x$ and $y^{\prime}$. Similarly, $\beta_{x}^{y}-\beta_{x}^{y^{\prime}}$ represents the rank of the homology groups which exist at $x$ and are destroyed between $y$ and $y^{\prime}$. Since $y$ is greater than $x$, any group existing at $x^{\prime}$ and destroyed between $y$ and $y^{\prime}$ also exists at $x$. More formally, we define surjective mappings 
$\Psi: W_{x}^{y} \rightarrow W_{x}^{y^{\prime}}$ and $\Psi^{\prime}: W_{x^{\prime}}^{y} \rightarrow W_{x^{\prime}}^{y^{\prime}}$ whose kernels represent the specified homology groups and show that the kernel of $\Psi^{\prime}$ is a subgroup of the kernel of $\Psi$.

As before, $x \leq y \leq y^{\prime}$. Define the mapping $\Psi: W_{x}^{y} \rightarrow W_{x}^{y^{\prime}}$ as follows. Let $h_{y}$ be an element of $W_{x}^{y} \subseteq H_{k}\left(F_{-\infty}^{y}\right)$. Since $h_{y}$ is in $W_{x}^{y}$, element $h_{y}$ is the image of some $h_{x} \in H_{k}\left(F_{-\infty}^{x}\right)$ under the mapping $H_{k}\left(F_{-\infty}^{x}\right) \rightarrow H_{k}\left(F_{-\infty}^{y}\right)$. Let $h_{y}^{\prime}$ be the image of $h_{y}$ under the mapping $H_{k}\left(F_{-\infty}^{y}\right) \rightarrow H_{k}\left(F_{-\infty}^{y^{\prime}}\right)$. The mappings are

$$
h_{x}^{\prime} \in H_{k}\left(F_{-\infty}^{x^{\prime}}\right) \rightarrow h_{x} \in H_{k}\left(F_{-\infty}^{x}\right) \rightarrow h_{y} \in H_{k}\left(F_{-\infty}^{y}\right) \rightarrow h_{y}^{\prime} \in H_{k}\left(F_{-\infty}^{y^{\prime}}\right) .
$$

The composition mapping $H_{k}\left(F_{-\infty}^{x}\right) \rightarrow H_{k}\left(F_{-\infty}^{y}\right) \rightarrow H_{k}\left(F_{-\infty}^{y^{\prime}}\right)$ takes $h_{x}$ to $h_{y}$ to $h_{y}^{\prime}$. Thus $h_{y}^{\prime}$ is in $W_{x}^{y^{\prime}}$. Define $\Psi\left(h_{y}\right)=h_{y}^{\prime}$.

We claim that $\Psi$ is surjective. Let $\hat{h}_{y}^{\prime}$ be an element of $W_{x}^{y^{\prime}}$. Since $\hat{h}_{y}^{\prime}$ is in $W_{x}^{y^{\prime}}$, element $\hat{h}_{y}^{\prime}$ is the image of some $\hat{h}_{x} \in H_{k}\left(F_{-\infty}^{x}\right)$ under the mapping $H_{k}\left(F_{-\infty}^{x}\right) \rightarrow H_{k}\left(F_{-\infty}^{y}\right)$. Let $\hat{h}_{y}$ be the image of $\hat{h}_{x}$ under the mapping $H_{k}\left(F_{-\infty}^{x}\right) \rightarrow H_{k}\left(F_{-\infty}^{y}\right)$. Element $\hat{h}_{y}$ is in $W_{x}^{y}$ and $\Psi\left(\hat{h}_{y}\right)$ equals $\hat{h}_{y}^{\prime}$ so $\Psi$ is surjective.

The difference $\beta_{x}^{y}-\beta_{x}^{y^{\prime}}$ is the difference between the rank of $W_{x}^{y}$ and $W_{x}^{y^{\prime}}$. Since $\Psi: W_{x}^{y} \rightarrow W_{x}^{y^{\prime}}$ is surjective, this difference is simply the rank of the kernel of $\Psi$.

Define the mapping $\Psi^{\prime}: W_{x^{\prime}}^{y} \rightarrow W_{x^{\prime}}^{y^{\prime}}$ in the same manner as $\Psi$. Note that $W_{x}^{y}$ and $W_{x^{\prime}}^{y}$ are subgroups of $H_{k}\left(F_{-\infty}^{y}\right)$. We claim that the kernel of $\Psi^{\prime}$ is a subgroup of the kernel of $\Psi$. Let $h_{y} \in W_{x^{\prime}}^{y} \subseteq H_{k}\left(F_{-\infty}^{y}\right)$ be an element of the kernel of $\Psi^{\prime}$. Since $h_{y}$ is an element of $W_{x^{\prime}}^{y}$, element $h_{y}$ is the image of some $h_{x}^{\prime} \in H_{k}\left(F_{-\infty}^{x^{\prime}}\right)$ under the mapping $H_{k}\left(F_{-\infty}^{x^{\prime}}\right) \rightarrow$ $H_{k}\left(F_{-\infty}^{y}\right)$. Let $h_{x}$ be the image of $h_{x}^{\prime}$ under the mapping $H_{k}\left(F_{-\infty}^{x^{\prime}}\right) \rightarrow H_{k}\left(F_{-\infty}^{x}\right)$. Since the mapping $H_{k}\left(F_{-\infty}^{x}\right) \rightarrow H_{k}\left(F_{-\infty}^{y}\right) \rightarrow H_{k}\left(F_{-\infty}^{y^{\prime}}\right)$ maps $h_{x}$ to $h_{y}$ to zero, element $h_{y}$ is in the kernel of $\Psi$ and the kernel of $\Psi^{\prime}$ is a subgroup of the kernel of $\Psi$. Since the ranks of the kernels of $\Psi$ and $\Psi^{\prime}$ are $\beta_{x}^{y}-\beta_{x}^{y^{\prime}}$ and $\beta_{x^{\prime}}^{y}-\beta_{x^{\prime}}^{y^{\prime}}$, respectively, it follows that $\beta_{x}^{y}-\beta_{x}^{y^{\prime}} \geq \beta_{x^{\prime}}^{y}-\beta_{x^{\prime}}^{y^{\prime}}$.

Since no critical points share a critical value, $\beta_{b-\epsilon}^{b-\epsilon}-\beta_{b+\epsilon}^{b+\epsilon} \leq 1$ and the mapping $H_{k}\left(F_{-\infty}^{b-\epsilon}\right) \rightarrow H_{k}\left(F_{-\infty}^{b+\epsilon}\right)$ is either an injection or a surjection. If $H_{k}\left(F_{-\infty}^{b-\epsilon}\right) \rightarrow H_{k}\left(F_{-\infty}^{b+\epsilon}\right)$ is an injection, then $\beta_{b-\epsilon}^{b+\epsilon}$ equals $\beta_{b-\epsilon}^{b-\epsilon}$ and so $\beta_{b-\epsilon}^{b-\epsilon}-\beta_{b-\epsilon}^{b+\epsilon}$ equals zero. If $H_{k}\left(F_{-\infty}^{b-\epsilon}\right) \rightarrow$ $H_{k}\left(F_{-\infty}^{b+\epsilon}\right)$ is a surjection, then $\beta_{b-\epsilon}^{b+\epsilon}$ equals $\beta_{b+\epsilon}^{b+\epsilon}$ and so $\beta_{b-\epsilon}^{b-\epsilon}-\beta_{b-\epsilon}^{b+\epsilon}=\beta_{b-\epsilon}^{b-\epsilon}-\beta_{b+\epsilon}^{b+\epsilon} \leq 1$. In both cases, $\beta_{b-\epsilon}^{b-\epsilon}-\beta_{b-\epsilon}^{b+\epsilon}$ is at most one. Thus for all $x<b-\epsilon$,

$$
\beta_{x}^{b-\epsilon}-\beta_{x}^{b+\epsilon} \leq \beta_{b-\epsilon}^{b-\epsilon}-\beta_{b-\epsilon}^{b+\epsilon} \leq 1 .
$$

In particular, $\beta_{a-\epsilon}^{b-\epsilon}-\beta_{a-\epsilon}^{b+\epsilon} \leq 1$ and $\beta_{a+\epsilon}^{b-\epsilon}-\beta_{a+\epsilon}^{b+\epsilon} \leq 1$.

By definition,

$$
\mu_{a}^{b}=\left(\beta_{a+\epsilon}^{b-\epsilon}-\beta_{a+\epsilon}^{b+\epsilon}\right)-\left(\beta_{a-\epsilon}^{b-\epsilon}-\beta_{a-\epsilon}^{b+\epsilon}\right) .
$$

Since $\beta_{a-\epsilon}^{b-\epsilon} \geq \beta_{a-\epsilon}^{b+\epsilon}$ and $\beta_{a-\epsilon}^{b-\epsilon}-\beta_{a-\epsilon}^{b+\epsilon} \leq 1$, the value $\beta_{a-\epsilon}^{b-\epsilon}-\beta_{a-\epsilon}^{b+\epsilon}$ is either zero or one. Similarly, the value $\beta_{a+\epsilon}^{b-\epsilon}-\beta_{a+\epsilon}^{b+\epsilon}$ is either zero or one.

Since $(a, b)$ is a pair of critical values, $\mu_{a}^{b}$ is positive. The only way $\mu_{a}^{b}$ can be positive is if $\beta_{a+\epsilon}^{b-\epsilon}-\beta_{a+\epsilon}^{b+\epsilon}$ equals one and $\beta_{a-\epsilon}^{b-\epsilon}-\beta_{a-\epsilon}^{b+\epsilon}$ equals zero. Substituting one and zero in the formula for $\mu_{a}^{b}$ shows that $\mu_{a}^{b}$ equals one. 
Since $\beta_{a-\epsilon}^{b-\epsilon}-\beta_{a-\epsilon}^{b+\epsilon}$ equals zero, $\beta_{a^{\prime}+\epsilon}^{b-\epsilon}-\beta_{a^{\prime}+\epsilon}^{b+\epsilon} \leq \beta_{a-\epsilon}^{b-\epsilon}-\beta_{a-\epsilon}^{b+\epsilon}=0$ must also be zero for all $a^{\prime}+\epsilon \leq a-\epsilon$. Thus, $\mu_{a^{\prime}}^{b}$ must be zero for all $a^{\prime}<a$. On the other hand, if $\mu_{a^{\prime}}^{b}$ equaled one for some $a^{\prime}>a$, then this argument would show that $\mu_{a}^{b}$ must equal zero. Thus for each $b$ there is at most one non-zero $\mu_{a}^{b}$.

Similarly, if $a^{\prime \prime} \leq a-\epsilon$, then $\beta_{a^{\prime \prime}}^{b-\epsilon}-\beta_{a^{\prime \prime}}^{b+\epsilon} \leq \beta_{a-\epsilon}^{b-\epsilon}-\beta_{a-\epsilon}^{b+\epsilon}$. Since $\beta_{a-\epsilon}^{b-\epsilon}-\beta_{a-\epsilon}^{b+\epsilon}$ equals zero, $\beta_{a^{\prime \prime}}^{b-\epsilon}-\beta_{a^{\prime \prime}}^{b+\epsilon}$ equals zero. If $a-\epsilon<a^{\prime \prime}<a$, then $\beta_{a^{\prime \prime}}^{b-\epsilon}$ equals $\beta_{a-\epsilon}^{b-\epsilon}$ and $\beta_{a^{\prime \prime}}^{b+\epsilon}$ equals $\beta_{a-\epsilon}^{b+\epsilon}$, so again $\beta_{a^{\prime \prime}}^{b-\epsilon}-\beta_{a^{\prime \prime}}^{b+\epsilon}$ equals zero.

Proof of Theorem 2. Let $(p, q)$ where $f(p)<f(q)$ is a pair of critical points with topological persistence $f(q)-f(p)$. By Lemma $15, \mu_{f(p)}^{f(q)}$ equals one. Let $k$ be the index of the homology groups $H_{k}$ used in determining the $\mu_{a}^{b}$. We wish to show that point $q$ has interval persistence $f(q)-f(p)$.

Part I: Show that the interval persistence of $q$ is at least $f(q)-f(p)$.

Let $a$ equal $f(p)$ and $b$ equal $f(q)$. By Lemma $15, \mu_{a}^{b}$ equals one. Since the persistent Betti numbers are non-negative, $\beta_{a+\epsilon}^{b-\epsilon}-\beta_{a+\epsilon}^{b+\epsilon}$ must equal one and $\beta_{a-\epsilon}^{b-\epsilon}-\beta_{a-\epsilon}^{b+\epsilon}$ must equal zero. Since $\left(\beta_{a+\epsilon}^{b-\epsilon}-\beta_{a+\epsilon}^{b+\epsilon}\right)$ equals one, there is some $h \in H_{k}\left(F_{-\infty}^{a+\epsilon}\right)$ which maps to zero under $H_{k}\left(F_{-\infty}^{a+\epsilon}\right) \rightarrow H_{k}\left(F_{-\infty}^{b+\epsilon}\right)$ but not under $H_{k}\left(F_{-\infty}^{a+\epsilon}\right) \rightarrow H_{k}\left(F_{-\infty}^{b-\epsilon}\right)$. Since the only critical value between $b-\epsilon$ and $b+\epsilon$ is at $b$, mapping $H_{k}\left(F_{-\infty}^{a+\epsilon}\right) \rightarrow H_{k}\left(F_{-\infty}^{b} \cup\right.$ $\left.f^{-1}(b)\right)$ sends $h$ to zero. Since $q$ is the only critical point with critical value $b$, mapping $H_{k}\left(F_{-\infty}^{a+\epsilon}\right) \rightarrow H_{k}\left(F_{-\infty}^{b} \cup\{q\}\right)$ sends $h$ to zero.

Let $a^{\prime}$ be any real between $a$ and $a+\epsilon$, i.e., $a<a^{\prime}<a+\epsilon$. We claim that there is some $\hat{h} \in H_{k}\left(f^{-1}\left(a^{\prime}\right)\right)$ whose image is $h$ under the mapping $H_{k}\left(f^{-1}\left(a^{\prime}\right)\right) \rightarrow H_{k}\left(F_{-\infty}^{a+\epsilon}\right)$ and which is destroyed at point $q$. Since $f$ is a Morse function, there is some $\epsilon^{\prime}$ such that $a<a^{\prime}-\epsilon^{\prime}<a^{\prime}<a^{\prime}+\epsilon^{\prime}<a+\epsilon$ and $f^{-1}\left(a^{\prime}\right)$ is a strong deformation retract of $N_{\epsilon^{\prime}}\left(f^{-1}\left(a^{\prime}\right)\right)$. Thus, $H_{k}\left(f^{-1}\left(a^{\prime}\right)\right) \rightarrow H_{k}\left(N_{\epsilon^{\prime}}\left(f^{-1}\left(a^{\prime}\right)\right)\right)$ is an isomorphism.

Since there are no critical values between $a$ and $a+\epsilon, H_{k}\left(F_{-\infty}^{a^{\prime}+\epsilon^{\prime}}\right) \rightarrow H_{k}\left(F_{-\infty}^{a+\epsilon}\right)$ is an isomorphism. Let $h^{\prime}$ be the element of $H_{k}\left(F_{-\infty}^{a^{\prime}+\epsilon^{\prime}}\right)$ whose image is $h \in H_{k}\left(F_{-\infty}^{a+\epsilon}\right)$ under this mapping. The mapping $H_{k}\left(F_{-\infty}^{a^{\prime}+\epsilon^{\prime}}\right) \rightarrow H_{k}\left(F_{-\infty}^{a^{\prime}+\epsilon^{\prime}}\right) \cup f^{-1}(b)$ takes $h^{\prime}$ to zero. The intersection of $H_{k}\left(F_{-\infty}^{a^{\prime}+\epsilon^{\prime}}\right)$ and $H_{k}\left(F_{a^{\prime}-\epsilon^{\prime}}^{b}\right) \cup f^{-1}(b)$ is $N_{\epsilon^{\prime}}\left(f^{-1}\left(a^{\prime}\right)\right)$ and their union is $F_{-\infty}^{b} \cup f^{-1}(b)$. By Corollary $8, h^{\prime}$ is the image of some $h^{\prime \prime} \in H_{k}\left(N_{\epsilon^{\prime}}\left(f^{-1}\left(a^{\prime}\right)\right)\right)$ under the mapping $H_{k}\left(N_{\epsilon^{\prime}}\left(f^{-1}\left(a^{\prime}\right)\right)\right) \rightarrow H_{k}\left(F_{-\infty}^{a^{\prime}+\epsilon^{\prime}}\right)$. Since the mapping $H_{k}\left(f^{-1}\left(a^{\prime}\right)\right) \rightarrow$ $H_{k}\left(N_{\epsilon^{\prime}}\left(f^{-1}\left(a^{\prime}\right)\right)\right)$ is an isomorphism, $h^{\prime \prime}$ is the image of some $\hat{h}$ under this mapping.

The mapping

$$
\begin{aligned}
H_{k}\left(f^{-1}\left(a^{\prime}\right)\right) \rightarrow H_{k}\left(N_{\epsilon^{\prime}}\left(f^{-1}\left(a^{\prime}\right)\right)\right) & \\
\downarrow & \\
H_{k}\left(F_{-\infty}^{a^{\prime}+\epsilon^{\prime}}\right) & \longrightarrow H_{k}\left(F_{-\infty}^{a+\epsilon}\right)
\end{aligned}
$$

takes $\hat{h}$ to $h^{\prime \prime}$ to $h^{\prime}$ to $h$. Since $h$ is destroyed by $q$, so is $\hat{h}$. Thus, for any $a^{\prime}$ between $a$ and $a+\epsilon$, some element of $H_{k}\left(f^{-1}\left(a^{\prime}\right)\right)$ is destroyed by $q$. Since the interval persistence of $q$ is

$$
\sup \left\{f(q)-a^{\prime}: q \text { destroys some non-zero } h \in H_{k}\left(f^{-1}\left(a^{\prime}\right)\right)\right\},
$$

and $\sup \left\{f(q)-a^{\prime}: a<a^{\prime}<a+\epsilon\right\}$ equals $f(q)-a$, the interval persistence is at least $f(q)-a=f(q)-f(p)$. 
Part II: Show that the interval persistence of $q$ is at most $f(q)-f(p)$.

Again let $a$ equal $f(p)$ and let $b$ equal $f(q)$. If the interval persistence of $q$ is more than $f(q)-f(p)$ then for some $a^{\prime}<a$, some non-zero $h \in H_{k}\left(f^{-1}\left(a^{\prime}\right)\right)$ is destroyed by $q$. Let $a^{\prime \prime}$ be a real between $a^{\prime}$ and $a$, i.e., $a^{\prime}<a^{\prime \prime}<a$. Let $h_{a}^{\prime}$ be the image of $h$ under the mapping $H_{k}\left(f^{-1}\left(a^{\prime}\right)\right) \rightarrow H_{k}\left(\underline{F}_{a^{\prime}}^{a^{\prime \prime}}\right)$. The mappings

$$
H_{k}\left(f^{-1}\left(a^{\prime}\right)\right) \rightarrow H_{k}\left(\underline{F}_{a^{\prime}}^{a^{\prime \prime}}\right) \rightarrow H_{k}\left(\underline{F}_{a^{\prime}}^{b}\right) \rightarrow H_{k}\left(\underline{F}_{a^{\prime}}^{b} \cup\{q\}\right)
$$

take $h$ to $h_{a}^{\prime}$ to some $h^{\prime} \in H_{k}\left(\underline{F}_{a^{\prime}}^{b}\right)$ to $0 \in H_{k}\left(\underline{F}_{a^{\prime}}^{b} \cup\{q\}\right)$. Since $h$ is destroyed by $q$, element $h^{\prime}$ is non-zero. Thus $h_{a}^{\prime}$ is non-zero and $h_{a}^{\prime}$ is also destroyed at $q$.

Consider the image $h_{a}^{\prime \prime}$ of $h_{a}^{\prime}$ under the mapping $H_{k}\left(F_{a^{\prime}}^{a^{\prime \prime}}\right) \rightarrow H_{k}\left(F_{-\infty}^{a^{\prime \prime}}\right)$. If $h_{a}^{\prime \prime}$ is nonzero, then $h_{a}^{\prime \prime}$ is destroyed at $q$. Thus, $\beta_{a^{\prime \prime}}^{b-\epsilon}-\beta_{a^{\prime \prime}}^{b+\epsilon}$ is positive. However, since $a^{\prime \prime}<a$, $\beta_{a^{\prime \prime}}^{b-\epsilon}-\beta_{a^{\prime \prime}}^{b+\epsilon}$ equals zero by Lemma 15 , a contradiction.

Now assume that $h_{a}^{\prime \prime}$ is zero. Since $\mu_{a}^{b}$ equals one, $H_{k}\left(F_{-\infty}^{b-\epsilon}\right) \rightarrow H_{k}\left(F_{-\infty}^{b+\epsilon}\right)$ is not an isomorphism. Since $f$ is a Morse function, $H_{j}\left(F_{-\infty}^{b-\epsilon}\right) \rightarrow H_{j}\left(F_{-\infty}^{b+\epsilon}\right)$ is an isomorphism for all $j \neq k$.

Since the image of $h_{a}^{\prime}$ under the mapping $H_{k}\left(F_{a^{\prime}}^{a^{\prime \prime}}\right) \rightarrow H_{k}\left(F_{-\infty}^{a^{\prime \prime}}\right)$ is $h_{a}^{\prime \prime}=0$ and the image of $h_{a}^{\prime}$ under the mapping $H_{k}\left(F_{a^{\prime}}^{a^{\prime \prime}}\right) \rightarrow H_{k}\left(F_{a^{\prime}}^{b+\epsilon}\right)$ is zero, the image of $h_{a}^{\prime}$ under the mapping $H_{k}\left(F_{a^{\prime}}^{a^{\prime \prime}}\right) \rightarrow H_{k}\left(F_{-\infty}^{a^{\prime \prime}}\right) \oplus H_{k}\left(F_{a^{\prime}}^{b+\epsilon}\right)$ is $0 \oplus 0$. Since the mapping

$$
H_{k+1}\left(F_{-\infty}^{b+\epsilon}\right) \rightarrow H_{k}\left(F_{a^{\prime}}^{a^{\prime \prime}}\right) \rightarrow H_{k}\left(F_{-\infty}^{a^{\prime \prime}}\right) \oplus H_{k}\left(F_{a^{\prime}}^{b+\epsilon}\right)
$$

is exact by Mayer-Vietoris, element $h_{a}^{\prime}$ must be in the image of some $h_{b} \in H_{k+1}\left(F_{-\infty}^{b+\epsilon}\right)$ under the mapping $H_{k+1}\left(F_{-\infty}^{b+\epsilon}\right) \rightarrow H_{k}\left(F_{a^{\prime}}^{a^{\prime \prime}}\right)$.

On the other hand, $H_{k+1}\left(F_{-\infty}^{b-\epsilon}\right) \rightarrow H_{k+1}\left(F_{-\infty}^{b+\epsilon}\right)$ is an isomorphism. Thus $h_{b}$ is the image of some $h_{b}^{\prime} \in H_{k+1}\left(F_{-\infty}^{b-\epsilon}\right)$. The composed mappings $H_{k+1}\left(F_{-\infty}^{b-\epsilon}\right) \rightarrow H_{k+1}\left(F_{-\infty}^{b+\epsilon}\right) \rightarrow$ $H_{k}\left(F_{a^{\prime}}^{a^{\prime \prime}}\right)$ take $h_{b}^{\prime}$ to $h_{b}$ to non-zero $h_{a}^{\prime}$. Since $h_{a}^{\prime}$ is destroyed by $q$, the image of $h_{a}^{\prime}$ under mapping $H_{k}\left(F_{a^{\prime}}^{a^{\prime \prime}}\right) \rightarrow H_{k}\left(F_{a^{\prime}}^{b-\epsilon}\right)$ is non-zero. Thus the image of $h_{a}^{\prime}$ under the mapping $H_{k}\left(F_{a^{\prime}}^{a^{\prime \prime}}\right) \rightarrow H_{k}\left(F_{a^{\prime}}^{b-\epsilon}\right) \oplus H_{k}\left(F_{-\infty}^{a^{\prime \prime}}\right)$ is non-zero. By Mayer-Vietoris, the mapping

$$
H_{k+1}\left(F_{-\infty}^{b-\epsilon}\right) \rightarrow H_{k}\left(F_{a^{\prime}}^{a^{\prime \prime}}\right) \rightarrow H_{k}\left(F_{-\infty}^{a^{\prime \prime}}\right) \oplus H_{k}\left(F_{a^{\prime}}^{b-\epsilon}\right)
$$

is exact. Since $h_{a}^{\prime}$ is in the image of $h_{b}^{\prime}$ under the mapping $H_{k+1}\left(F_{-\infty}^{b-\epsilon}\right) \rightarrow H_{k}\left(F_{a^{\prime}}^{a^{\prime \prime}}\right)$, the image of $h_{a}^{\prime}$ under the mapping $H_{k}\left(F_{a^{\prime}}^{a^{\prime \prime}}\right) \rightarrow H_{k}\left(F_{a^{\prime}}^{b-\epsilon}\right) \oplus H_{k}\left(F_{-\infty}^{a^{\prime \prime}}\right)$ is zero, a contradiction. Thus $h_{a}^{\prime \prime}$ cannot be zero and the interval persistence of $q$ is at most $f(q)-f(p)$.

\section{Appendix D. Properties of LR Maps}

Lemma 16 says that if $f$ is LR, then we can replace $\{x: a \leq f(x) \leq b\}$ by suitably chosen small neighborhoods without changing its homology.

Lemma 16. If continuous function $f: \Omega \rightarrow \mathbb{R}$ is $L R$, then for every $a, b \in \mathbb{R}$ where $a<b$, there exists an $\epsilon_{0}$ such that, for all $\epsilon<\epsilon_{0}$, set $\{x: a \leq f(x) \leq b\}$ is a strong deformation retract of $\{x: a \leq f(x)<b+\epsilon\}$ and set $\{x: a \leq f(x)<b\}$ is a strong deformation retract of $\{x: a-\epsilon<f(x)<b\}$. 
Proof. Assume function $f: \Omega \rightarrow \mathbb{R}$ is LR. Choose $\epsilon_{1}>0$ such that $f^{-1}(a)$ is a strong deformation retract of $\{x: a-\epsilon<f(x)<a+\epsilon\}$ for all $\epsilon<\epsilon_{1}$. Similarly, choose $\epsilon_{2}>0$ such that $f^{-1}(b)$ is a strong deformation retract of $\{x: b-\epsilon<f(x)<b+\epsilon\}$ for all $\epsilon<\epsilon_{2}$. Let $\epsilon_{0}$ be the minimum of $\epsilon_{1}$ and $\epsilon_{2}$ and $(b-a) / 2$.

For any $\epsilon \leq \epsilon_{0}$, let $\varphi_{\epsilon}^{a}$ be the mapping from $\{a: a-\epsilon<f(x)<a+\epsilon\} \times I$ to $f^{-1}(a)$ representing the strong deformation retract of $\{a: a-\epsilon<x<a+\epsilon\}$ to $f^{-1}(a)$. Let $\varphi_{\epsilon}^{b}$ be the mapping from $\{b: b-\epsilon<f(x)<b+\epsilon\} \times I$ to $f^{-1}(b)$ representing the strong deformation retract of $\{b: b-\epsilon<x<b+\epsilon\}$ to $f^{-1}(b)$. Define

$$
\varphi_{\epsilon}(x, t)= \begin{cases}\varphi_{\epsilon}^{a}(x, t) & \text { for } \quad a-\epsilon<x<a, \\ x & \text { for } \quad a \leq x \leq b, \\ \varphi_{\epsilon}^{b}(x, t) & \text { for } \quad b<x<b+\epsilon .\end{cases}
$$

$\varphi_{\epsilon}$ is constant on $\{x: a \leq x \leq b\}$ and continuously deforms $\{x: a-\epsilon<x<a\}$ and $\{x: b<x<b+\epsilon\}$ onto $\{x: a \leq x \leq b\}$. Thus $\{x: a \leq f(x) \leq b\}$ is a strong deformation retract of $\{x: a \leq f(x)<b+\epsilon\}$ and $\{x: a \leq f(x)<b\}$ is a strong deformation retract of $\{x: a-\epsilon<f(x)<b\}$.

Let non-zero $h \in H_{k}\left(f^{-1}(a)\right)$ be destroyed by $\underline{F}_{a}^{\infty}$. If $f$ is LR, then $h$ is destroyed by $\{x: a \leq f(x) \leq b\}$ for some $b \geq a$. Equivalently, the image of $h$ in $H_{k}\left(F_{a}^{b}\right)$ is destroyed by $f^{-1}(b)$.

Lemma 17. Let $f: \Omega \rightarrow \mathbb{R}$ be a continuous LR function. For any non-zero $h \in$ $H_{k}\left(f^{-1}(a)\right)$, if $\underline{F}_{a}^{\infty}$ destroys $h$, then for some $b \geq a$, the image of $h$ under the mapping $H_{k}\left(f^{-1}(a)\right) \rightarrow H_{k}\left(F_{a}^{b}\right)$ is destroyed by $f^{-1}(b)$.

Proof. Since $H_{k}\left(f^{-1}(a)\right) \rightarrow H_{k}\left(\underline{F}_{a}^{\infty}\right)$ sends $h$ to zero, element $h$ is the boundary of some chain $C \subseteq \underline{F}_{a}^{\infty}$. Chain $C$ is compact. (See p. 71 of [12].) Thus $\{f(x): x \in C\}$ is compact and has a maximum value $b^{\prime}$. Since $C \subseteq \underline{F}_{a}^{b^{\prime}}$, the mapping $H_{k}\left(f^{-1}(a)\right) \rightarrow$ $H_{k}\left(\underline{F}_{a}^{b^{\prime}}\right)$ sends $h$ to zero.

Let $b$ equal $\inf \left\{\tilde{b}: H_{k}\left(f^{-1}(a)\right) \rightarrow H_{k}\left(\underline{F}_{a}^{\tilde{b}}\right)\right.$ sends $h$ to zero $\}$. Note that $b \leq b^{\prime}$. Since $f$ is LR, $H_{k}\left(f^{-1}(a)\right)$ is isomorphic to $H_{k}\left(F_{a-\epsilon}^{a+\epsilon}\right)$ for sufficiently small $\epsilon$ and thus $H_{k}\left(f^{-1}(a)\right) \rightarrow H_{k}\left(\underline{F}_{a}^{a+\epsilon}\right)$ does not send $h$ to zero. Thus $b$ is strictly greater than $a$.

Let $h^{\prime}$ be the image of $h$ under the mapping $H_{k}\left(f^{-1}(a)\right) \rightarrow H_{k}\left(\underline{F}_{a}^{b}\right)$. If $h^{\prime}$ were zero, then $h$ would be the boundary of some chain $C^{\prime} \subseteq \underline{F}_{a}^{b}$. Since $C^{\prime}$ is compact, chain $C^{\prime}$ would also be a subset of $\underline{F}_{a}^{\tilde{b}}$ for some $\tilde{b}<b$, contradicting the choice of $b$. Thus $h^{\prime}$ is non-zero.

We show that $h^{\prime}$ is destroyed by $f^{-1}(b)$. Since $f$ is LR, there is some $\epsilon_{0}>0$ such that $H_{k}\left(\underline{F}_{a}^{b} \cup f^{-1}(b)\right)$ is isomorphic to $H_{k}\left(\underline{F}_{a}^{b+\epsilon}\right)$ for all $\epsilon \leq \epsilon_{0}$. If $H_{k}\left(\underline{F}_{a}^{b}\right) \rightarrow$ $H_{k}\left(\underline{F}_{a}^{b} \cup f^{-1}(b)\right)$ does not map $h^{\prime}$ to zero, then $H_{k}\left(\underline{F}_{a}^{b}\right) \rightarrow H_{k}\left(\underline{F}_{a}^{b+\epsilon}\right)$ does not map $h^{\prime}$ to zero for all $\epsilon \leq \epsilon_{0}$, and $b$ does not equal $\inf \left\{\tilde{b}: H_{k}\left(f^{-1}(b)\right) \rightarrow H_{k}\left(\underline{F}_{a}^{\tilde{b}}\right)\right.$ sends $h$ to zero $\}$. Thus, $H_{k}\left(\underline{F}_{a}^{b}\right) \rightarrow H_{k}\left(\underline{F}_{a}^{b} \cup f^{-1}(b)\right)$ maps $h^{\prime}$ to zero and $h^{\prime}$ is destroyed by $f^{-1}(b)$.

The proof of Lemma 2 follows from Lemma 17. 
Proof of Lemma 2. By Lemma 17, there exists some $b>a$ such that the image $h^{\prime}$ of $h$ under the mapping $H_{k}\left(f^{-1}(a)\right) \rightarrow H_{k}\left(F_{a}^{b}\right)$ is destroyed by $f^{-1}(b)$. Since $f$ is point destructible, there is some point $q \in f^{-1}(b)$ which destroys $h^{\prime}$ and $h$.

Proof of Lemma 4. Let $b$ equal $f(q)$. The intersection of $\underline{F}_{a_{1}}^{b}$ and $X \cup\{q\}$ is $X=\{x \in$ $\Omega: a_{2} \leq g(x)$ and $\left.f(x)<f(q)\right\}$, and their union is $\underline{F}_{a_{1}}^{b} \cup\{q\}$. By Corollary $8, h$ is the image of some $h_{x} \in H_{k}(X)$ under the mapping $H_{k}(X) \rightarrow H_{k}\left(\underline{F}_{a_{1}}^{b}\right)$ and $h$ is destroyed by $q$.

We now prove that $h$ is the image of some $h_{2} \in H_{k}\left(g^{-1}\left(a_{2}\right)\right)$. Since $g$ is LR, there exists some $\epsilon_{1}>0$ such that $H_{k}\left(g^{-1}\left(a_{2}\right)\right) \rightarrow H_{k}\left(N_{\epsilon^{\prime}}\left(g^{-1}\left(a_{2}\right)\right)\right)$ is an isomorphism for all $\epsilon^{\prime} \leq$ $\epsilon_{1}$. By Lemma 16, there exists some $\epsilon_{2}>0$ such that $H_{k}(X) \rightarrow H_{k}\left(X \cup N_{\epsilon^{\prime}}\left(g^{-1}\left(a_{2}\right)\right)\right)$ is an isomorphism for all $\epsilon^{\prime} \leq \epsilon_{2}$. Since $g^{-1}\left(a_{2}\right) \subseteq F_{a_{1}}^{b}$, there is some $\epsilon_{3}$ such that $N_{\epsilon_{3}}\left(g^{-1}\left(a_{2}\right)\right) \subseteq F_{a_{1}}^{b}$. Let $\epsilon$ be the smaller of $\epsilon_{1}, \epsilon_{2}$, and $\epsilon_{3}$.

Let $Y$ equal $N_{\epsilon}\left(g^{-1}\left(a_{2}\right)\right)$. Let $Z=\left\{x \in \Omega: a_{2}-\epsilon<g(x)\right.$ and $\left.f(x)<f(q)\right\}$ and $Z^{\prime}=\left\{x \in \Omega: a_{1} \leq f(x)\right.$ and $\left.g(x)<a_{2}+\epsilon\right\}$. The following commutative diagram gives the relevant mappings between homology groups:

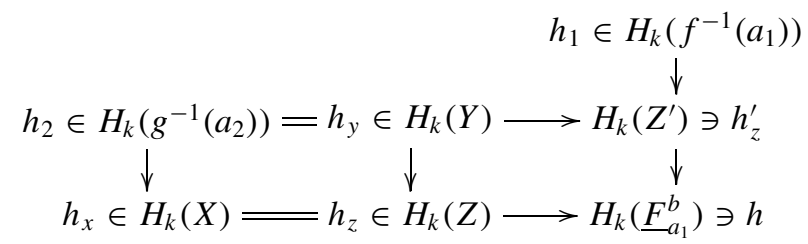

Let $h_{z}$ be the image of $h_{x}$ under the mapping $H_{k}(X) \rightarrow H_{k}(Z)$. Let $h_{z}^{\prime}$ be the image of $h_{1}$ under the mapping $H_{k}\left(f^{-1}\left(a_{1}\right)\right) \rightarrow H_{k}\left(Z^{\prime}\right)$. Element $h$ is the image of $h_{x} \in H_{k}(X)$ and $h_{1} \in H_{k}\left(f^{-1}\left(a_{1}\right)\right)$ and so is the image of both $h_{z}$ and $h_{z}^{\prime}$ under the respective mappings $H_{k}(Z) \rightarrow H_{k}(X)$ and $H_{k}\left(Z^{\prime}\right) \rightarrow H_{k}(X)$. By Corollary 10, there is some $h_{y} \in H_{k}(Y)$ such that $H_{k}(Y) \rightarrow H_{k}(Z)$ maps $h_{y}$ to $h_{z}$ and $H_{k}(Y) \rightarrow$ $H_{k}\left(Z^{\prime}\right)$ maps $h_{y}$ to $h_{z}^{\prime}$. Since $H_{k}\left(g^{-1}\left(a_{2}\right)\right) \rightarrow H_{k}(Y)$ is an isomorphism, there is some $h_{2} \in H_{k}\left(g^{-1}\left(a_{2}\right)\right)$ whose image is $h_{y}$ under the mapping $H_{k}\left(g^{-1}\left(a_{2}\right)\right) \rightarrow H_{k}(Y)$. The mapping $H_{k}\left(g^{-1}\left(a_{2}\right)\right) \rightarrow H_{k}(Y) \rightarrow H_{k}(Z) \rightarrow H_{k}\left(F_{a_{1}}^{b}\right)$ takes $h_{2}$ to $h_{y}$ to $h_{z}$ to $h$.

Proof of Lemma 3. Set $g$ equal to $f$ and apply Lemma 4.

Finally, we prove that piecewise linear functions on compact spaces are LR.

Lemma 18. A piecewise linear function $f: \Omega \rightarrow \mathbb{R}$ on the compact space $\Omega$ is $L R$.

Proof. By definition, $\Omega$ has a finite triangulation $\mathcal{T}$ such that $f$ is linear on each simplex in $\mathcal{T}$. Fix $a \in \mathbb{R}$. Since $\mathcal{T}$ has a finite number of simplices, there is some $\epsilon$ such that $\{x: a-\epsilon \leq x<a\}$ and $\{x: a<x \leq a+\epsilon\}$ do not contain any vertices of $\mathcal{T}$. Let $\Omega^{\prime}$ equal $\{x \in \Omega: a-\epsilon \leq x \leq a+\epsilon\}$. Let $\mathcal{T}^{\prime}$ be the triangulation of $\Omega^{\prime}$ induced by intersecting the simplices of $\mathcal{T}$ by $\{x \in \Omega: a-\epsilon \leq x \leq a\}$ and $\{x \in \Omega: a \leq x \leq a+\epsilon\}$ and subdividing the resulting convex polytopes without adding any new vertices. Note that the vertices of $\mathcal{T}^{\prime}$ all lie on $f^{-1}(a-\epsilon)$ or $f^{-1}(a)$ or $f^{-1}(a+\epsilon)$. 
Let $V$ be the vertices of $\mathcal{T}^{\prime}$. Each vertex $v \in V$ which does not lie on $f^{-1}(a)$ lies on a unique edge $e_{v}$ of $\mathcal{T}$. Since sets $\{x: a-\epsilon<x<a\}$ and $\{x: a<x<a+\epsilon\}$ do not contain any vertices of $\mathcal{T}$, edge $e_{v}$ intersects $f^{-1}(a)$. Since $f$ is linear, $e_{v}$ intersects $f^{-1}(a)$ at only one point. Let $\tilde{v}$ be the point $e_{v} \cap f^{-1}(a)$. For each vertex $v \in V$ which lies on $f^{-1}(a)$, let $\tilde{v}$ equal $v$.

Define the map $\varphi: V \times[0,1] \rightarrow N_{\epsilon}(a)$ as $\varphi(v, s)=(1-s) v+s \tilde{v}$. Note that this map is constant on all points in $f^{-1}(a)$.

Extend map $\varphi$ linearly over each simplex in $\mathcal{T}^{\prime}$ forming a map over $\Omega^{\prime} \times[0,1]$. More specifically, each point $x \in \Omega^{\prime}$ lies in some simplex $t^{\prime} \in \mathcal{T}^{\prime}$ whose vertices are $V \cap t^{\prime}$. Point $x$ is a convex combination, $\sum_{v \in V \cap t^{\prime}} \alpha_{v} v$, of the points $V \cap t^{\prime}$. Define $\varphi(x, s)=\sum_{v \in V \cap t^{\prime}} \alpha_{v} \varphi(v, s)$. Since $\varphi$ is constant on all vertices in $f^{-1}(a)$, map $\varphi$ is also constant on all simplices in $f^{-1}(a)$ and thus constant on $f^{-1}(a)$. Thus, $\varphi(x, 0)=x$ and $\varphi(x, 1) \in f^{-1}(a)$ for all $x \in \Omega$ and $\varphi(x, s)=x$ for all $x \in f^{-1}(a)$ and $f^{-1}(a)$ is a strong deformation retract of $\Omega^{\prime}$. Restricting $\varphi$ to the open set $N_{\epsilon}\left(f^{-1}(a)\right) \subseteq \Omega^{\prime}$ shows that $f^{-1}(a)$ is a strong deformation retract of $N_{\epsilon}\left(f^{-1}(a)\right)$. Thus, $f$ is locally retractible.

\section{Appendix E. Maxima}

The following homology lemma is used in the proof of the pairwise disjointness of $(\gamma, \infty)$-neighborhoods of local maxima.

Lemma 19. Let $M$ be a connected, oriented d-manifold with non-empty boundary. Let $D_{1}, D_{2}, \ldots, D_{k}$ be the connected components of $\partial M$ with orientation inherited from $M$. If $a_{1} D_{1}+a_{2} D_{2}+\cdots+a_{k} D_{k}$ generate the zero element of $H_{d-1}(M)$, then $a_{1}=a_{2}=\cdots=a_{k}$.

Proof. The sequence $H_{d}(M) \rightarrow H_{d}(M, \partial M) \rightarrow H_{d-1}(\partial M) \rightarrow H_{d-1}(M)$ is exact [9, Theorem 2.16, p. 117]. Since the boundary of $M$ is not empty, the homology group $H_{d}(M)$ is zero. The homology group of $H_{d}(M, \partial M)$ is $\mathbb{G}$, the ground ring of the homology group. The map $H_{d}(M, \partial M) \rightarrow H_{d-1}(\partial M)$ is the connecting homomorphism. It maps $\mathbb{G}$ to $h \in H_{d-1}(\partial M)$ which is generated by $D_{1}+D_{2}+\cdots+D_{k}$. Since the mapping is exact, the image of $\mathbb{G} h$ under the mapping $H_{d-1}(\partial M) \rightarrow H_{d-1}(M)$ is zero. Moreover, only elements in $\mathbb{G} h$ map to zero. Thus, if $a_{1} D_{1}+a_{2} D_{2}+\cdots+a_{k} D_{k}$ generate the zero element of $H_{d-1}(M)$, then $a_{1} D_{1}+a_{2} D_{2}+\cdots+a_{k} D_{k}$ must generate an element of $\mathbb{G} h$ in $H_{d-1}(\partial M)$ and so $a_{1}=a_{2}=\cdots=a_{k}$.

\section{References}

1. P. K. Agarwal, H. Edelsbrunner, J. Harer, and Y. Wang. Extreme elevation on a 2-manifold. In SCG '04: Proceedings of the Twentieth Annual Symposium on Computational Geometry, pages 357-365. ACM Press, New York, 2004.

2. G. Carlsson, A. Collins, L. Guibas, and A. Zomorodian. Persistence barcodes for shapes. In Proc. 2nd Sympos. Geometry Process., pages 127-138, 2004. 
3. F. Chazal and A. Lieutier. Weak feature size and persistent homology: computing homology of solids in $\mathbb{R}^{n}$ from noisy data samples. In Proc. 21st Annu. ACM Sympos. Comput. Geom., pages 255-262, 2005.

4. D. Cohen-Steiner, H. Edelsbrunner, and J. Harer. Stability of persistence diagrams. In Proc. 21st Annu. ACM Sympos. Comput. Geom., pages 263-271, 2005.

5. D. Cohen-Steiner, H. Edelsbrunner, and D. Morozov. Vines and vineyards by updating persistence in linear time. In Proc. 22nd Annu. ACM Sympos. Comput. Geom., pages 119-126, 2006.

6. T. K. Dey, J. Giesen, and S. Goswami. Shape segmentation and matching with flow discretization. In Proc. 8th Workshop on Algorithms Data Structures, pages 25-36, 2003.

7. H. Edelsbrunner, J. Harer, and A. Zomorodian. Hierarchical Morse complexes for piecewise linear 2manifolds. Discrete Comput. Geom., 30:87-107, 2003.

8. H. Edelsbrunner, D. Letscher, and A. Zomorodian. Topological persistence and simplification. Discrete Comput. Geom., 28:511-533, 2002.

9. A. Hatcher. Algebraic Topology. Cambridge University Press, Cambridge, 2002.

10. M. W. Hirsch. Differential Topology. Springer, New York, 1976.

11. J. Milnor. Morse Theory. Princeton University Press, Princeton, NJ, 1963.

12. J. J. Rotman. An Introduction to Algebraic Topology. Springer-Verlag, Berlin, 1988.

13. A. Zomorodian and G. Carlsson. Computing perisistent homology. Discrete Comput. Geom., 33:249-274, 2005.

Received August 28, 2006, and in revised form April 16, 2007. Online publication August 24, 2007. 